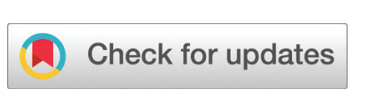

Cite this: Food Funct., 2021, 12, 8100

\title{
Human milk oligosaccharides and non-digestible carbohydrates prevent adhesion of specific pathogens via modulating glycosylation or inflammatory genes in intestinal epithelial cells
}

\author{
Chunli Kong, (D) *a,b Martin Beukema, (D) ${ }^{\mathrm{b}}$ Min Wang, ${ }^{\mathrm{c}}$ Bart J. de Haan ${ }^{\mathrm{b}}$ and \\ Paul de Vos ${ }^{\mathrm{b}}$
}

\begin{abstract}
Human milk oligosaccharides (hMOs) and non-digestible carbohydrates (NDCs) are known to inhibit the adhesion of pathogens to the gut epithelium, but the mechanisms involved are not well understood. Here, the effects of 2'-FL, 3-FL, DP3-DP10, DP10-DP60 and DP30-DP60 inulins and DM7, DM55 and DM69 pectins were studied on pathogen adhesion to Caco-2 cells. As the growth phase influences virulence, E. coli ET8, E. coli LMG5862, E. coli O119, E. coli WA321, and S. enterica subsp. enterica LMG07233 from both log and stationary phases were tested. Specificity for enteric pathogens was tested by including the lung pathogen $K$. pneumoniae LMG20218. Expression of the cell membrane glycosylation genes of galectin and glycocalyx and inflammatory genes was studied in the presence and absence of 2'-FL or NDCs. Inhibition of pathogen adhesion was observed for 2'-FL, inulins, and pectins. Pre-incubation with 2'-FL downregulated ICAMI, and pectins modified the glycosylation genes. In contrast, $K$. pneumoniae LMG20218 downregulated the inflammatory genes, but these were restored by pre-incubation with pectins, which reduced the adhesion of $K$. pneumoniae LMG20218. In addition, DM69 pectin significantly upregulated the inflammatory genes. $2^{\prime}-\mathrm{FL}$ and pectins but not inulins inhibited pathogen adhesion to the gut epithelial Caco-2 cells through changing the cell membrane glycosylation and inflammatory genes, but the effects were molecule-, pathogen-, and growth phase-dependent.
\end{abstract}

Received 22nd March 2021, Accepted 21st June 2021

DOI: $10.1039 / \mathrm{d} 1 \mathrm{fo} 00872 \mathrm{~b}$

rsc.li/food-function carbohydrates (NDCs) such as inulins, galacto-oligosaccharides and pectins are applied. ${ }^{5,6}$ In recent years, some synthetic hMOs have been applied in infant formula as a new emerging technique allowing for cost-effective production. ${ }^{7}$

An important function of hMOs and NDCs in infant formula is the reduction of pathogen infection. ${ }^{7}$ A possible mechanism by which this is accomplished is through direct modulation of the intestinal epithelial glycosylation receptors. ${ }^{8,9}$ The glycocalyx layer covering the intestinal epithelium is known to be an anchoring point for pathogens and has been reported to change in response to molecules such as hMOs. ${ }^{10}$ The intestinal epithelial glycocalyx is built up of a core protein of glypicans linked to the cell membrane and further branched with glycosaminoglycan side chains of hyaluronic acid (HA) or heparan sulfate (HS). ${ }^{11}$ HA and HS play an important role in the binding of pathogens and other antigens in the gut lumen. ${ }^{12,13}$ Within the glycocalyx layer, galectins form a large family of lectins with affinity for $N$-acetyllactosamine residues and regulate the inflammatory responses by mediating protein-glycan and protein-protein interactions. ${ }^{14}$ Among the 15 identified galectins in the glycocalyx, galectin-1 is a 'proto-type' galectin with one carbohydrate recognition domain (CRD) and it facilitates the secretion of anti-inflammatory cytokines. ${ }^{14}$ 
Galectin-9 is a 'tandem repeat-type' galectin with two CRDs, ${ }^{15}$ and was reported to be a target for the treatment of intestinal inflammatory diseases. ${ }^{16}$

During the pathogenesis of infection of gut epithelial cells, many other molecules are also involved in the inflammatory responses. ${ }^{17,18}$ Intercellular adhesion molecule-1 (ICAM-1) is such a molecule. It is a trans-membrane glycoprotein in epithelial cells, which is reported to be highly expressed during inflammation $^{18}$ and to recruit leukocytes. ${ }^{19}$ The chemokine CXC motif ligands and human beta defensin have also been reported to play an active role in the development of intestinal epithelial inflammation. ${ }^{17,20}$ For example, higher incidence of necrotizing enterocolitis is associated with increased CXCL1 expression in mice. ${ }^{21}$ Infection and inflammation usually occur after interaction between the gut epithelium and specific, but to be identified, bacterial virulence and adhesion molecules on pathogens. ${ }^{22,23}$ It is well known that expression of these adhesion receptors depends on the growth phase of the pathogen. ${ }^{24}$ Pathogens from the stationary phase have a thicker peptidoglycan layer, which contribute to biofilm formation and stronger resistance to environmental stresses compared to pathogens from the log phase. ${ }^{24,25}$ As in the intestine, pathogens may be in both phases, depending on the stage of the infection, ${ }^{26}$ thus it is important to study the anti-adhesion effects of hMOs and NDCs after treating the gut epithelium with pathogens from both the log and stationary phases.

As there is limited information available about how hMOs and NDCs modulate gut epithelial cells and how this might contribute to the prevention of pathogen adhesion, we pre-incubated gut epithelial Caco-2 cells with different hMOs and NDCs and subsequently exposed them to different bacterial pathogens. Anti-adhesion effects were tested for the gut pathogens E. coli ET8, E. coli LMG5862, E. coli O119, E. coli WA321, Salmonella enterica subsp. enterica LMG07233, and a lung pathogen Klebsiella pneumoniae LMG20218. They were tested after harvesting from both $\log$ and stationary growth phases. Effects of hMOs and NDCs as well as of the pathogens were tested on the gene expression in Caco-2 cells of galectin-1 (LGALS1), galectin-9 (LGALS9), glycocalyx-related molecules (glypican 1 [GPC1], hyaluronan synthase 3 [HAS3], exostosin glycosyltransferase 1 [EXT1], EXT2), ICAM-1, chemokine CXC motif ligands (CXCL1, CXCL2, CXCL3 and CCL20), and antimicrobial peptide human defensin $\beta-1$ (DEFB1). We tested the effects of the most abundant hMOs in mother's milk, i.e. 2'-fucosyllactose (2'-FL) and 3-FL. Also, we tested and compared the effects of NDCs which are often used as substitutes in infant formula including inulins with different degrees of polymerization (DP3-DP10, DP10DP60 and DP30-60) and pectins with different degrees of methylation (DM7, DM55 and DM69).

\section{Materials and methods}

\subsection{Carbohydrates}

The hMOs 2'-FL and 3-FL were obtained from Elicityl (France). Chicory based inulins with different degrees of polymerization, i.e. highly soluble Frutafit® CLR inulin of DP3-DP10 and moderately soluble Frutafit ${ }^{\circledR}$ TEX! inulins of DP10-DP60 and DP30-DP60, were provided by Sensus (the Netherlands). Lemon pectins with different degrees of methylation including DM7, DM55, and DM69 were from CP Kelco (Denmark). All carbohydrates were dissolved with antibiotic-free cell culture medium into $2 \mathrm{mg} \mathrm{ml}^{-1}$.

\subsection{Cell culture}

Human intestinal epithelial Caco-2 cells (HTB-37, ATCC) at passage number 15-20 were cultured in Dulbecco's modified Eagle's medium (Lonza, Verviers, Belgium), supplemented with $10 \%(\mathrm{v} / \mathrm{v}$, heat deactivated) fetal calf serum (Thermo Scientific, Breda, the Netherlands), 2.5\% (v/v) HEPES, $50 \mathrm{U}$ $\mathrm{ml}^{-1}$ penicillin, $50 \mu \mathrm{g} \mathrm{ml} \mathrm{m}^{-1}$ streptomycin, and $1 \%(\mathrm{v} / \mathrm{v})$ nonessential amino acid (Sigma-Aldrich, Zwijndrecht, the Netherlands). The cells were routinely cultured in a $5 \% \mathrm{CO}_{2}$ incubator at $37{ }^{\circ} \mathrm{C}$. To ensure the confluency and consistency of Caco-2 cells, the transepithelial electrical resistance (TEER) value was determined in transwell plates using an electric cell substrate impedance sensing (ECIS) system (Applied Biophysics). A total of $1.5 \times 10^{4}$ cells per well were seeded into the 24-well plates and cultured for 21 days until a TEER value of $300 \pm 10 \Omega \mathrm{cm}^{2}$ was reached.

\subsection{Enteric pathogens and culture}

Enteric pathogens including Escherichia (E.) coli ET8, E. coli LMG5862, E. coli O119, E. coli WA321, Salmonella (S.) enterica subsp. enterica LMG07233, and Klebsiella (K.) pneumoniae LMG20218 (ATCC) were tested. Bacterial strains were recovered from glycerol stocks in brain heart infusion (BHI) broth. After recovery, all strains were plated on BHI agar plates. A single colony from the agar plates was inoculated to BHI broth for overnight culture at $37{ }^{\circ} \mathrm{C}$, and sub-cultured until either the logarithmic (log) phase or the stationary phase was reached. The cultures were centrifuged at $3000 \mathrm{~g}$ for $10 \mathrm{~min}$ and the pellets were resuspended in PBS. The optical density (OD) value was adjusted to $\mathrm{OD}_{600}=0.6 \pm 0.02$ (approx. $1 \times 10^{9} \mathrm{CFU}$ $\mathrm{ml}^{-1}$ ) and the pellets were washed once in PBS before inoculation.

\subsection{Anti-adhesion assay}

Caco-2 cells were pre-incubated with carbohydrates $(2 \mathrm{mg}$ $\mathrm{ml}^{-1}$ ) for 2 hours. The same volume of prepared pathogens from the log or stationary phase was inoculated for another 1 hour (E. coli LMG5862) or 2 hours (all other strains) of incubation. E. coli LMG5862 needed 1 hour incubation, because longer infection times destroyed the integrity of the Caco- 2 cell monolayer. Cells pre-incubated without carbohydrates served as the control. After infection, Caco- 2 cells were gently washed three times with PBS to remove the non-adherent bacteria. The adherent bacteria were released with $200 \mu$ l of $0.1 \%$ Triton X-100 for 10-15 $\mathrm{min}$ and subjected to serial dilutions. The drop-plate method was applied for quantification. ${ }^{27}$ The results were expressed as relative adhesion compared to the controls. 


\subsection{RNA isolation and reverse transcription}

The results of the anti-adhesion assays led to selection of 2 FL, inulins, and pectins for gene expression analysis. As described above in the anti-adhesion assay, after 21 days of culture, Caco- 2 cells were pre-incubated with 2 '-FL, inulins, and pectins for $2 \mathrm{~h}$. After infection with or without the pathogens, Caco-2 monolayers were lysed with TRIzol reagent (Life Technologies, Carlsbad, USA). Total RNA isolation was performed according to the manufacturer's instructions. SuperScript II reverse transcriptase (Invitrogen, Breda, the Netherlands) was used to synthesize cDNA for performing quantitative PCR.

\subsection{Gene expression}

LGALS1, LGALS9, HAS3, EXT1, EXT2, GPC1, ICAM1, CXCL1, CXCL2, CXCL3, CCL20, and DEFB1 expressions were quantified using $\mathrm{SYBR}{ }^{\circledR}$ Green Real-time PCR Master Mix (Roche, Basel, Switzerland). Reactions were carried out in 384-well PCR plates (Thermo Scientific, UK) using the ViiA7 Real-Time PCR system (Applied Biosystems). Expression levels were normalized to the housekeeping gene $G A P D H$. The $2^{-\Delta \Delta \mathrm{Ct}}$ method was used for the calculation of the fold changes in gene expression versus the group without pathogen infection. All the primers were synthesized by Sigma-Aldrich as listed in Table $1 .{ }^{28-36}$

\subsection{Statistical analysis}

All data were analyzed with GraphPad Prism 6 software (GraphPad Software, Inc., San Diego, USA). The KolmogorovSmirnov test was performed to confirm the normal distribution of the data. The results were expressed as mean \pm SD of at least five experiments. All the data were analyzed using Kruskal-Wallis one-way ANOVA followed by Dunn's multiple comparison test. Significant difference was defined as $p<0.05$ ${ }^{*} p<0.05,{ }^{* *} p<0.01,{ }^{* * *} p<0.001$ and $\left.{ }^{* * * *} p<0.0001\right) . p<0.1$ was defined as a trend. ${ }^{37}$

\section{Results}

HMOs and NDCs have been suggested to modulate gut epithelial cell membrane bound receptors, ${ }^{38,39}$ and by that prevent pathogen infections. It is unknown which and how these molecules can modulate gut epithelial cells such as Caco-2 and which pathogens might be prevented from binding to these cells. To this end, we tested the anti-adhesion effects of the hMOs 2'-FL and 3-FL, inulins with DP3-DP10, DP10DP60, and DP30-DP60, and pectins with DM7, DM55, and DM69. We tested the adhesion of the enteric pathogens $E$. coli ET8, E. coli LMG5862, E. coli O119, E. coli WA321, and $S$. enterica subsp. enterica LMG07233. One lung pathogen K. pneumoniae LMG20218 was tested to determine the gut pathogen-specific effects. Pathogens from both log and stationary phases were tested as the bacteria-host interaction may differ with the bacterial growth phase. We pre-incubated hMOs and NDCs with Caco- 2 cells before pathogen infection to determine possible prevention of pathogen adhesion after modulation of the intestinal epithelial cells by the molecules.

\subsection{2 '-FL induced anti-adhesion effects on the pathogens from the log phase}

Log phase. We pre-incubated Caco-2 cells with 2'-FL and 3-FL for $2 \mathrm{~h}$ before they were infected with the pathogens harvested from the log phase (Fig. 1A-F). We observed a $23.05 \%$ inhibition of E. coli $\mathrm{O} 119$ adherence to Caco-2 cells with 2 '-FL ( $p<$ 0.05 , Fig. 1C). Pre-incubating Caco-2 cells with 3-FL had no effect on the adhesion of the tested pathogens to Caco- 2 cells.

Stationary phase. Pre-incubating Caco-2 cells with hMOs did not show any inhibition of adhesion of the tested pathogens from the stationary growth phase (Fig. 1G-L).

\subsection{Inulins induced anti-adhesion effects on pathogens from both log and stationary phases in a DP dependent manner}

Log phase. Pre-incubating Caco-2 cells with inulins resulted in anti-adhesion effects on specific pathogens in a DP dependent

Table 1 Primer sequences used for real-time $\mathrm{qPCR}^{28-36}$

\begin{tabular}{|c|c|c|}
\hline Primer $^{a}$ & Forward & Reverse \\
\hline LGALS1 & 5'-TGCAACAGCAAGGACGGC & 5'-CACCTCTGCAACACTTCCA \\
\hline LGALS9 & 5'-CTTTCATCACCACСATTCTG & 5'-ATGTGGAACCTCTGAGCACTG \\
\hline HAS3 & 5'-TATACCGCGCGCTCCAA & 5'-GCCACTCCCGGAAGTAAGACT \\
\hline EXT1 & 5'-CATAGGCGATGAGAGATTGT & 5'-CAAGAATTGTGTCTGCTGTC \\
\hline EXT2 & 5'-GGCTACGATGTCAGCATTCCTG & 5'-GGCTTCTAGGTCCTCTCTGTAC \\
\hline GPC1 & 5'-TATTGCCGAAATGTGCTCAAGGGC & 5'-ATGACACTCTCCACACCCGATGTA \\
\hline ICAM1 & 5'-GGCCTCAGTCAGTGTGA & 5'-AACCCCATTCAGCGTCA \\
\hline$C X C L 1$ & 5'-AGTCATAGCCACACTCAAGAATGG & 5'-GATGCAGGATTGAGGCAAGC \\
\hline CXCL2 & 5'-CTCAAGAATGGGCAGAAAGC & 5'-AAACACATTAGGCGCAATCC \\
\hline CXCL3 & 5'-GCAGGGAATTCACCTCAAGA & 5'-GGTGCTCCCCTTGTTCAGTA \\
\hline CCL20 & 5'-GTGGCTTTTCTGGAATGGAA & 5'-GACAAGTCCAGTGAGGCACA \\
\hline$D E F B 1$ & 5'-CCTTCTGCTGTTTACTCTCTGC & 5'-GAATAGAGACATTGCCCTCCAC \\
\hline$G A P D H$ & 5'-AAGATCATCAGCAATGCCTCCTGC & 5'-ATGGACTGTGGTCATGAGTCCTTC \\
\hline
\end{tabular}

${ }^{a}$ LGALS1, galectin 1; LGALS9, galectin 9; HAS3, hyaluronan synthase 3; EXT, exostosin glycosyltransferase; GPC1, glypican 1; ICAM1, intercellular adhesion molecule 1; CXCL/CCL, chemokine; DEFB1, defensin beta 1 . 

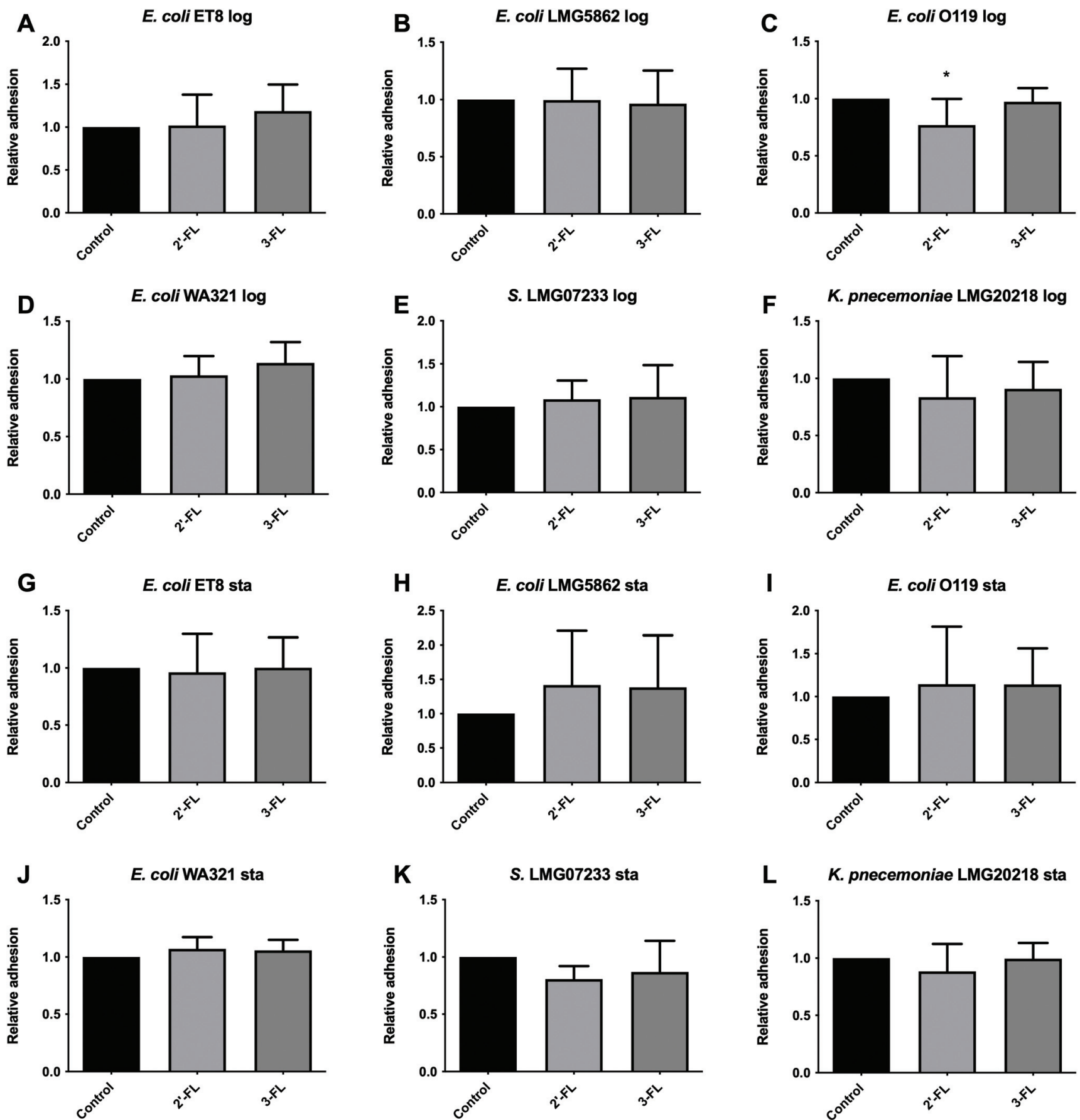

Fig. 1 Anti-adhesion effects induced by hMOs on pathogens harvested from the log and stationary growth phases to Caco-2 cells. Caco-2 cells were cultured for 21 days until confluency. hMOs of 2'-FL and 3-FL were pre-incubated with Caco-2 cells for $2 \mathrm{~h}$ before infection with the pathogens harvested from the log phase. These were E. coli ET8 (A), E. coli LMG5862 (B), E. coli O119 (C), E. coli WA321 (D), S. LMG07233 (E), and K. pneumoniae LMG20218 (F) or harvested from the stationary phase, i.e. E. coli ET8 (G), E. coli LMG5862 (H), E. coli O119 (I), E. coli WA321 (J), S. LMG07233 (K), and K. pneumoniae LMG20218 (L) for another $2 \mathrm{~h}$. After infection, the total colony forming units (CFUs) of the pathogens adhered to Caco- 2 cells were determined by a drop-plating method. The group without hMOs served as the control. The adhesion of pathogens was normalized to the control (the control was set as 1). All data were expressed as mean \pm SD from five experiments. Statistical comparison was tested using one-way ANOVA $\left({ }^{*} p<0.05\right)$. Log and Sta stand for logarithmic and stationary stages of pathogen growth, respectively.

manner (Fig. 2A-F). DP10-DP60 inulin significantly reduced the adhesion of $E$. coli ET8 by $37.40 \%$ ( $p<0.05$, Fig. $2 \mathrm{~A}$ ), and DP30-DP60 inulin significantly reduced the adhesion of $E$. coli
O119 in the log phase by $26.25 \%$ ( $p<0.05$, Fig. 2C). DP3-DP10 and DP10-DP60 inulins reduced the adhesion of K. pneumoniae LMG20218 (Fig. $2 \mathrm{~F}$ ) by $29.44 \%$ and $29.45 \%$, 


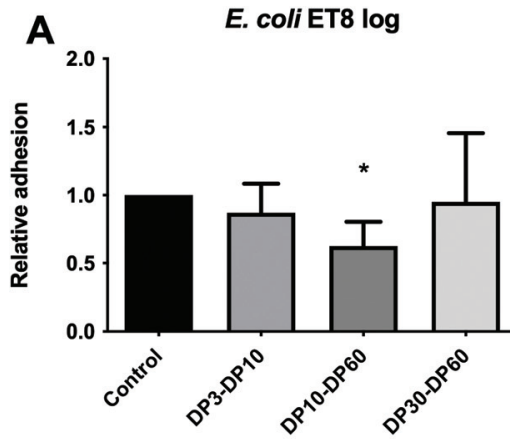

D

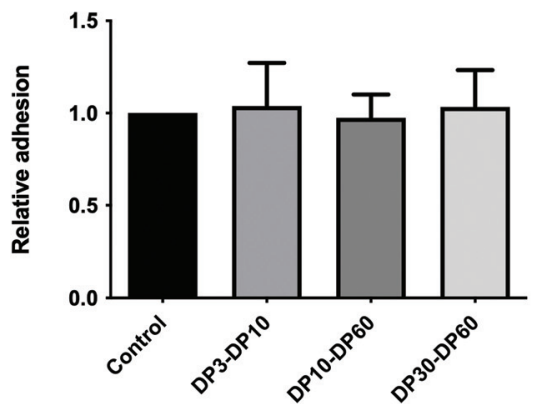

G

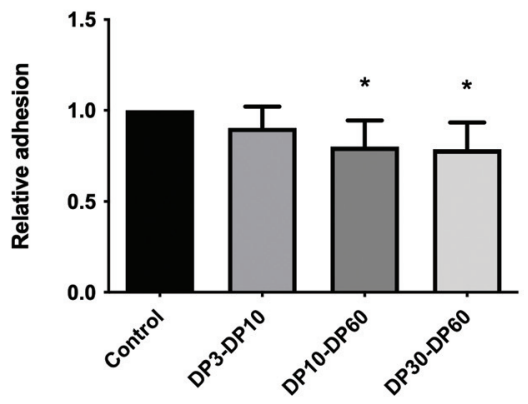

J

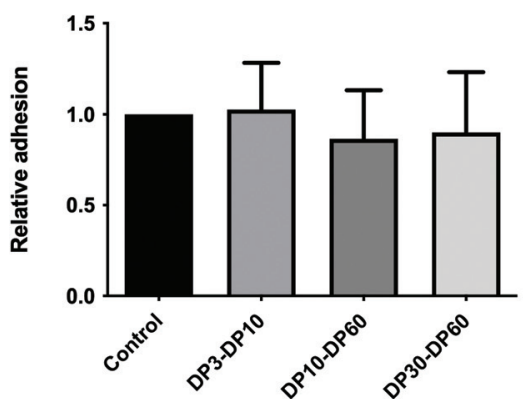

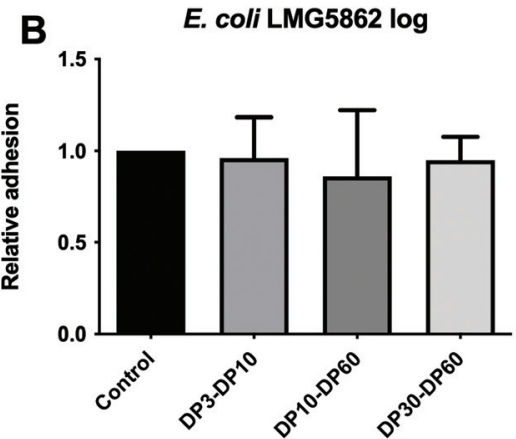

E

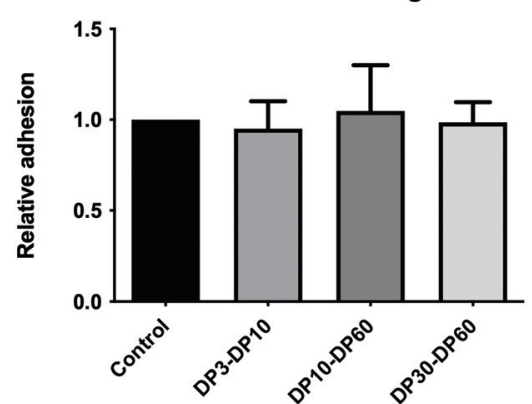

H

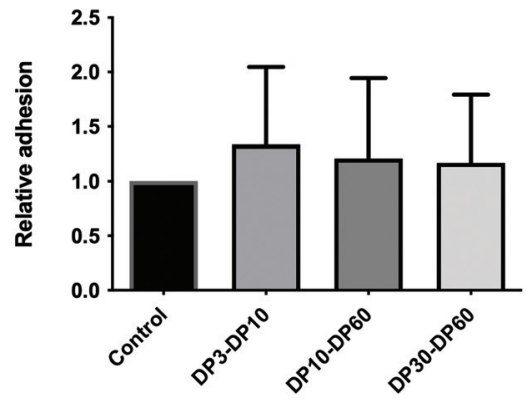

K

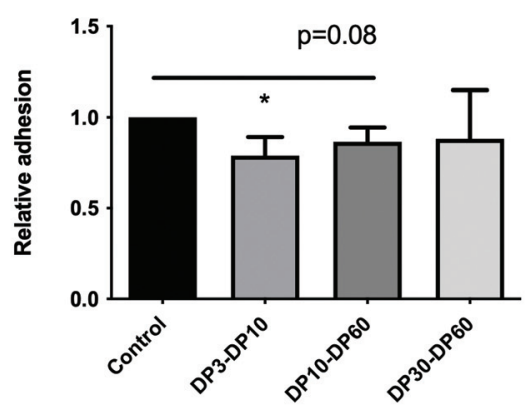

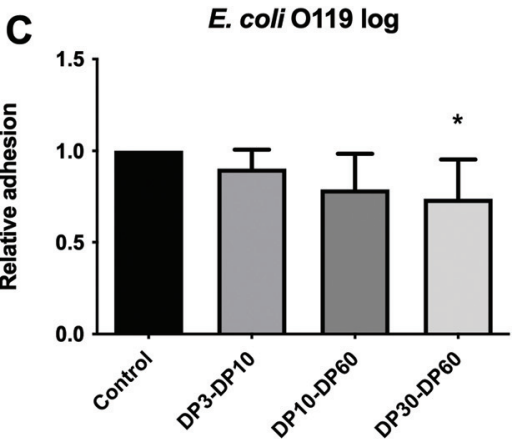

F K. pnecemoniae LMG20218 log

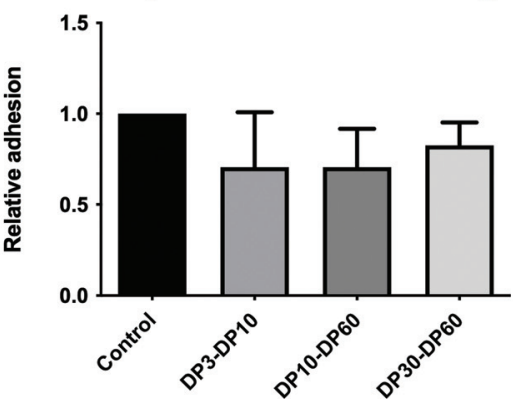

I

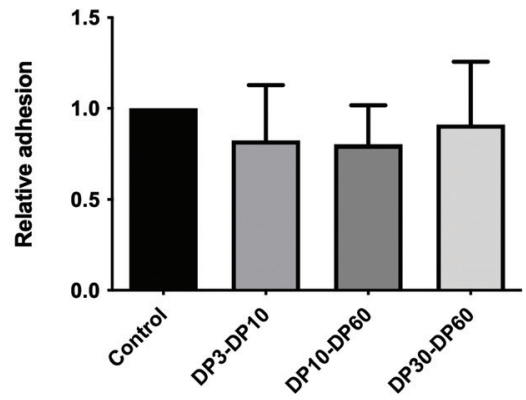

L

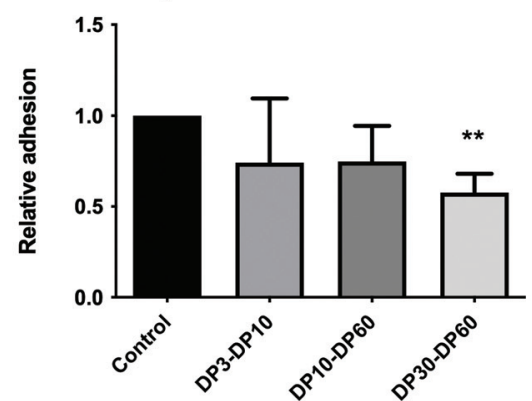

Fig. 2 Anti-adhesion effects induced by inulins on pathogens harvested from the log and stationary growth phases to Caco-2 cells. Caco-2 cells were cultured for 21 days until confluency. Inulins of DP3-DP10, DP10-DP60, and DP30-DP60 were pre-incubated with Caco-2 cells for $2 \mathrm{~h}$ before infection with the pathogens harvested from the log phase, i.e. E. coli ET8 (A), E. coli LMG5862 (B), E. coli O119 (C), E. coli WA321 (D), S. LMG07233 (E), and K. pneumoniae LMG20218 (F) or harvested from the stationary phase, i.e. E. coli ET8 (G), E. coli LMG5862 (H), E. coli O119 (I), E. coli WA321 (J), S. LMG07233 (K), and K. pneumoniae LMG20218 (L) for another $2 \mathrm{~h}$. After infection, the total colony forming units (CFUs) of the pathogens adhered to Caco-2 cells were determined by a drop-plating method. The group without inulins served as the control. The adhesion of pathogens was normalized to the control (the control was set as 1). All data were expressed as mean \pm SD from five experiments. Statistical comparison was tested using one-way ANOVA ${ }^{*} p<0.05$ and $p<0.1$ were considered a trend). Log and Sta stand for logarithmic and stationary stages of the pathogen growth, respectively. 
respectively, but this did not reach statistical significance. Other pathogens were not influenced by the pre-incubation of inulins with Caco-2 cells.

Stationary phase. Pre-incubating Caco-2 cells with inulins induced anti-adhesion effects in the stationary phase also in a DP dependent manner. DP3-DP10 inulin selectively inhibited the adhesion of $S$. enterica subsp. enterica LMG07233 by $21.01 \%(p<0.05$, Fig. 2K). DP10-DP60 inulin significantly reduced the adhesion of $E$ coli ET 8 in the stationary phase by $19.91 \%$ ( $p<0.05$, Fig. $2 \mathrm{G})$ and had the tendency to inhibit the adhesion of $S$. enterica subsp. enterica LMG07233 (Fig. 2K) by 13.59\%. DP30-DP60 inulin had anti-adhesion effects on both E. coli ET8 ( $p<0.05$, Fig. $2 \mathrm{G})$ and $K$. pneumoniae LMG20218 ( $p$ $<0.01$, Fig. 2L), which were $21.44 \%$ inhibition for $E$. coli ET8 and $42.34 \%$ for K. pneumoniae LMG20218.

\subsection{Pectins induced anti-adhesion effects on the pathogens from both log and stationary phases in a DM dependent manner, and DM55 and DM69 pectins specifically increased the adhesion of $E$. coli WA321 from the stationary phase}

Log phase. Pre-incubating Caco-2 cells with pectins had a DM dependent anti-adhesive effect on the pathogens. DM7 pectin significantly inhibited the adhesion of E. coli O119 $(p<0.01$, Fig. 3C) and $K$. pneumoniae LMG20218 ( $p<0.05$, Fig. 3F) by $35.74 \%$ and $38.96 \%$, respectively. DM55 pectin significantly reduced the adhesion of $E$. coli ET8 $(p<0.01$, Fig. 3A) by $47.35 \%$, E. coli LMG5862 ( $p<0.01$, Fig. $3 \mathrm{~B}$ ) by $43.41 \%$, and K. pneumoniae LMG20218 ( $p<0.05$, Fig. 3 F) by $43.37 \%$. DM69 pectin selectively suppressed the adhesion of E. coli LMG5862 $(p<0.05$, Fig. 3B) to Caco- 2 cells by $35.96 \%$.

Stationary phase. Pre-incubating Caco-2 cells with DM55 pectin significantly reduced the adhesion of $E$. coli ET8 by $40.59 \%$ ( $p<0.01$, Fig. 3G). In contrast, DM55 and DM69 pectins significantly increased the adhesion of $E$. coli WA321 ( $p<0.05$, Fig. 3J) by $92.58 \%$ and $84.83 \%$, respectively. All of the tested pectins significantly inhibited the adhesion of the lung pathogen $\mathrm{K}$. pneumoniae LMG20218 to Caco-2 cells, with a reduction of $34.71 \%$ by DM7 pectin $(p<0.05$, Fig. $3 \mathrm{~L})$, with $54.99 \%$ by DM55 pectin $(p<0.01$, Fig. $3 \mathrm{~L}$ ), and with $42.52 \%$ by DM69 pectin ( $p<0.05$, Fig. $3 \mathrm{~L})$.

\subsection{2'-FL and pectins but not inulins modulated the cell membrane glycosylation genes and inflammation associated genes in intestinal epithelial Caco- 2 cells}

As we found that 2 '-FL, inulins, and pectins differently influenced the adhesion of pathogens to Caco-2 cells, we next determined the gene expression of cell membrane bound components that might be involved in pathogen adhesion and infection. We included the pathogens from either the log or the stationary phase whose adhesion to Caco-2 cells was significantly influenced. To this end, the expressions of cell membrane glycosylation genes, such as those of galectins (LGALS1 and LGALS9) and glycocalyx (HAS3, EXT1, EXT2 and GPC1), and inflammation associated genes, such as those of cell adhesion, chemotaxis target (ICAM1, CXCL1, CXCL2, CXCL3 and $C C L 20)$ and anti-microbial peptide (DEFB1), were studied.
3.4.1. E. coli ET8. Log phase. E. coli ET8 from the log phase did not influence the gene expressions of LGALS1, LGALS9, EXT1, EXT2, and GPC1 (Fig. 4A, B, D-F), but significantly downregulated HAS3 (Fig. 4C, $p<0.01$ ), which decreased by $34.25 \%$ compared to Caco- 2 cells without infection. E. coli ET8 infection significantly upregulated adhesion and the chemotaxis target genes, i.e. CXCL2 and CCL20, and showed a tendency to upregulate CXCL1 and CXCL3, with an increase of 1.65-fold (Fig. 4I, $p<$ 0.05), 2.25-fold (Fig. $4 \mathrm{~K}, p<0.01$ ), 2.17-fold (Fig. $4 \mathrm{H}, p=0.060$ ), and 2.92-fold (Fig. 4J, $p=0.066$ ), respectively. However, pre-incubating the cells with either DP10-DP60 inulin or DM55 pectin did not influence the above genes with $E$. coli ET8 infection.

Stationary phase. E. coli ET8 from the stationary phase also significantly downregulated the glycosylation gene $H A S 3$, with a reduction of $36.86 \%$ (Fig. 5C, $p<0.01$ ), but not LGALS1, LGALS9, $E X T 1, E X T 2$, and GPC1 in Caco-2 cells. Pre-incubating the cells with DP10-DP60 inulin, DP30-DP60 inulin, or DM55 pectin did not influence the above genes with $E$. coli ET8 infection.

3.4.2. E. coli LMG5862. Log phase. With the infection of E. coli LMG5862 from the log phase, glycosylation genes including HAS3, EXT1, EXT2, and GPC1 were significantly downregulated, with a reduction of $58.54 \%$ (Fig. 6C, $P<$ 0.0001 ), $48.94 \%$ (Fig. 6D, $P<0.0001$ ), 37.56\% (Fig. 6E, $P<$ 0.01 ), and $54.47 \%$ (Fig. 6F, $P<0.0001$ ), respectively. Adhesion and chemotaxis target genes and the anti-microbial peptide gene were not influenced with $E$. coli LMG5862 infection. Pre-incubating Caco- 2 cells with DM69 pectin but not DM55 pectin significantly upregulated the glycosylation gene HAS3 (Fig. 6C, $p<0.05$ ), with an increase of $20.67 \%$, compared to the cells exposed to $E$. coli LMG5862. Adhesion and chemotaxis target genes including ICAM1, CXCL1, CXCL3, and CCL20 were also significantly upregulated by DM69 pectin, with a 1.09-fold (Fig. 6G, $p<0.0001$ ), a 79.47-fold (Fig. 6H, $p<0.01$ ), a 47.87-fold (Fig. 6J, $p<0.01$ ), and a 41.26-fold (Fig. 6K, $p<0.0001$ ) increase, respectively.

3.4.3. E. coli 0119. Log phase. Similar to what we observed in E. coli LMG5862 (Fig. 6), E. coli O119 from the log phase also significantly downregulated the glycosylation gene $H A S 3$, and showed a tendency to downregulate GPC1, with a reduction of $42.20 \%$ (Fig. 7C, $P<0.001$ ) and 25.56\% (Fig. 7F, $P=0.099$ ), respectively. E. coli $\mathrm{O} 119$ infection significantly upregulated the adhesion and chemotaxis target genes of ICAM1, CXCL1, and CCL20, and a tendency with CXCL3, which was as high as 1.71fold (Fig. 7G, $p<0.05$ ), 29.84-fold (Fig. 7H, $p<0.05$ ), 9.33-fold (Fig. 7K, $p<0.05$ ), and 23.59-fold (Fig. 7J, $p=0.066$ ) higher than the gene expression without E. coli $\mathrm{O} 119$ infection, respectively. Pre-incubating the cells with 2'-FL specifically significantly downregulated the expression of ICAM1 (Fig. 7G, $p<0.05$ ). However, pre-incubating the cells with DP30-DP60 inulin or DM7 pectin did not influence the above genes with $E$. coli O119 infection.

3.4.4. E. coli WA321. Stationary phase. E. coli WA321 from the stationary phase showed a tendency to downregulate the glycosylation gene $H A S 3$, with a reduction of $41.87 \%$ (Fig. $8 \mathrm{C}, p=$ 0.099). DM69 pectin was observed to have a tendency to upregulate HAS3 (Fig. 8C, $p=0.071$ ). DM69 pectin dramatically upregulated all of the adhesion and chemotaxis target genes of ICAM1, $C X C L 1, C X C L 2, C X C L 3$, and CCL20, with an increase of up to 


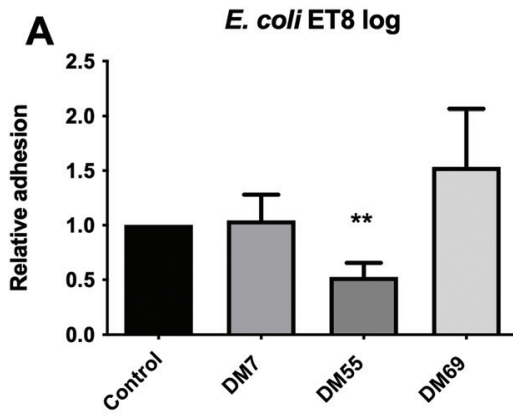

D E. coli WA321 log

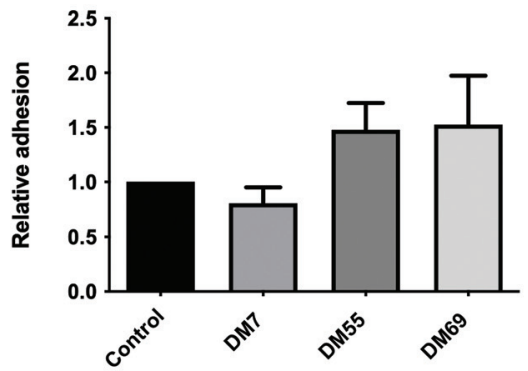

G

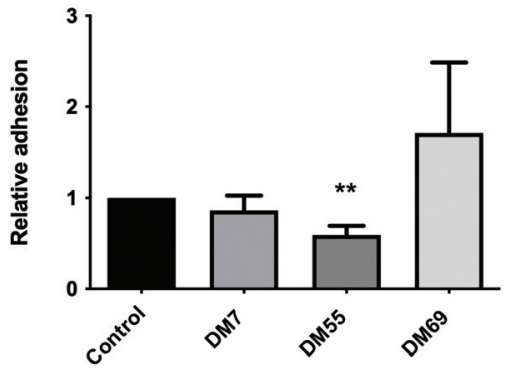

J

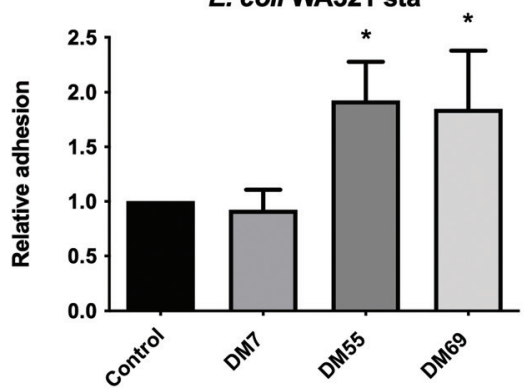

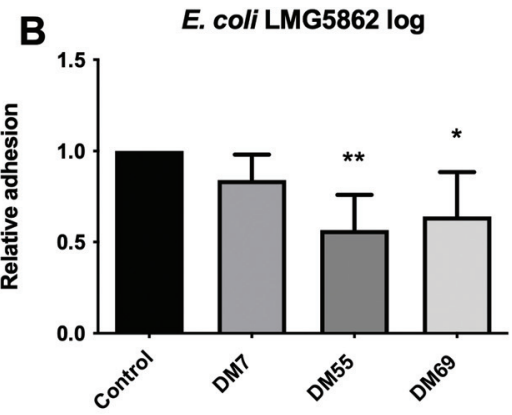

E

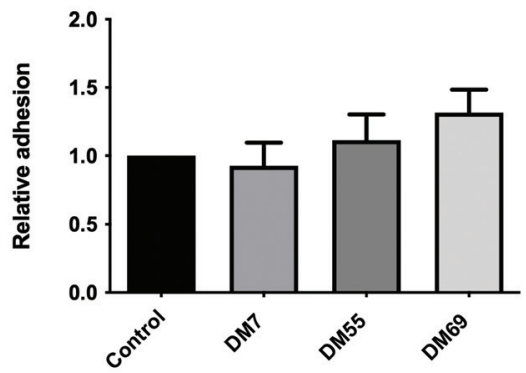

H

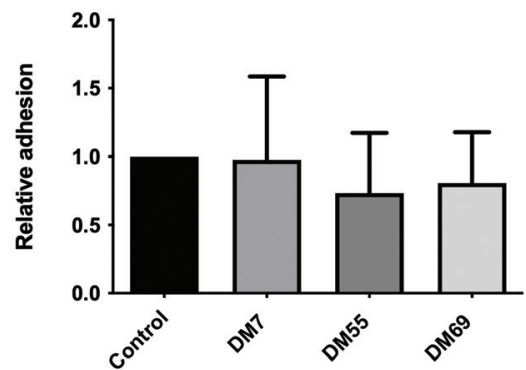

K

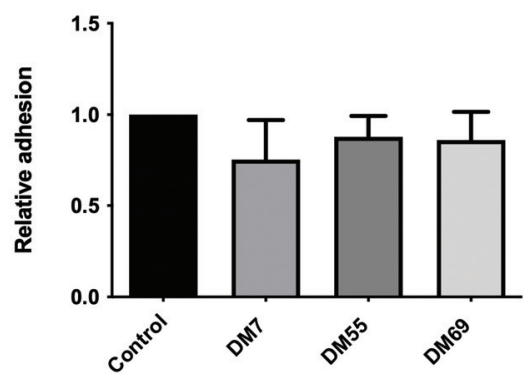

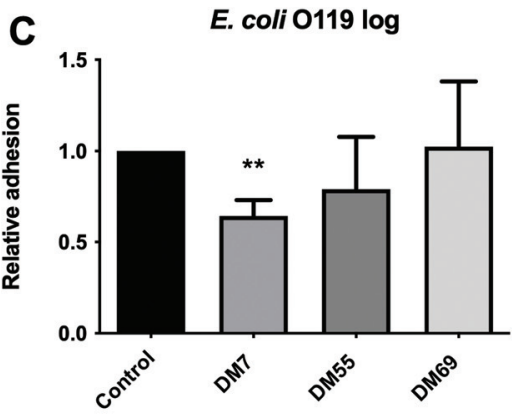

F K. pnecemoniae LMG20218 log

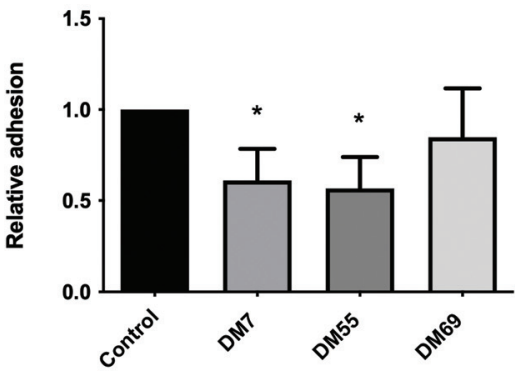

I

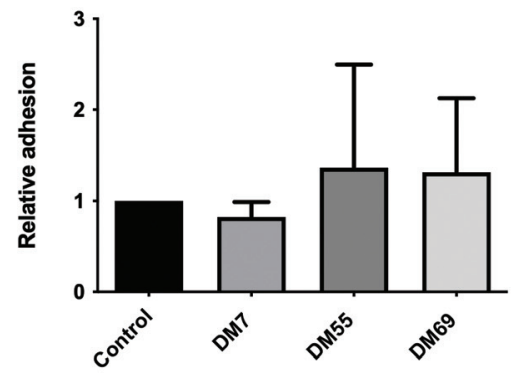

L

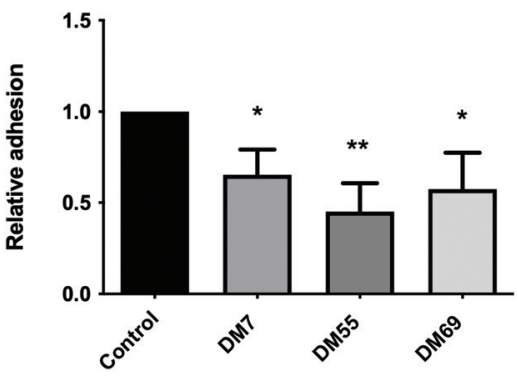

Fig. 3 Anti-adhesion effects induced by pectins on pathogens harvested from the log and stationary growth phases to Caco-2 cells. Caco-2 cells were cultured for 21 days until confluency. Pectins of DM7, DM55, and DM69 were pre-incubated with Caco-2 cells for $2 \mathrm{~h}$ before infection with the pathogens harvested from the log phase, i.e. E. coli ET8 (A), E. coli LMG5862 (B), E. coli O119 (C), E. coli WA321 (D), S. LMG07233 (E), and K. pneumoniae LMG20218 (F) or harvested from the stationary phase, i.e. E. coli ET8 (G), E. coli LMG5862 (H), E. coli O119 (I), E. coli WA321 (J), S. LMG07233 (K), and K. pneumoniae LMG20218 (L) for another $2 \mathrm{~h}$. After infection, the total colony forming units (CFUs) of the pathogens adhered to Caco- 2 cells were determined by a drop-plating method. The group without pectins served as the control. The adhesion of pathogens was normalized to the control (the control was set as 1). All data were expressed as mean \pm SD from five experiments. Statistical comparison was tested using one-way ANOVA ( ${ }^{*} p<0.05$ and ${ }^{* *} p<0.01$ ). Log and Sta stand for logarithmic and stationary stages of pathogen growth, respectively. 
A

LGALS1

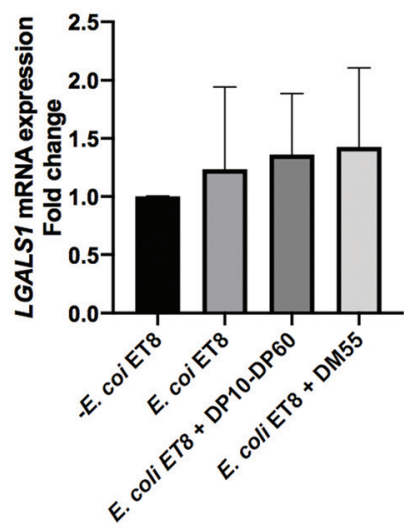

E

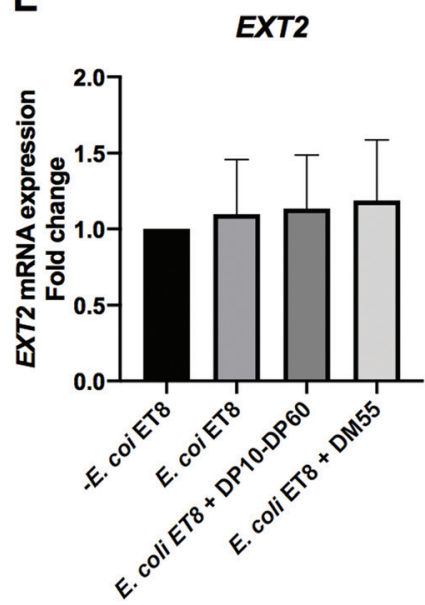

I

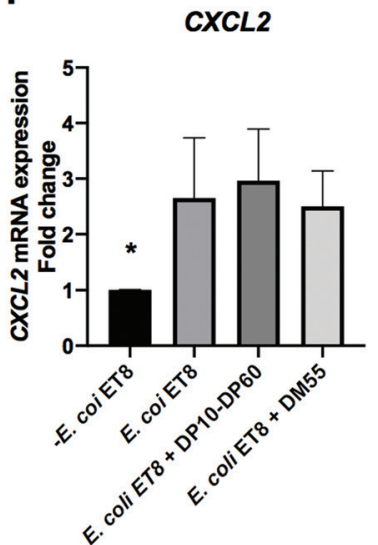

B

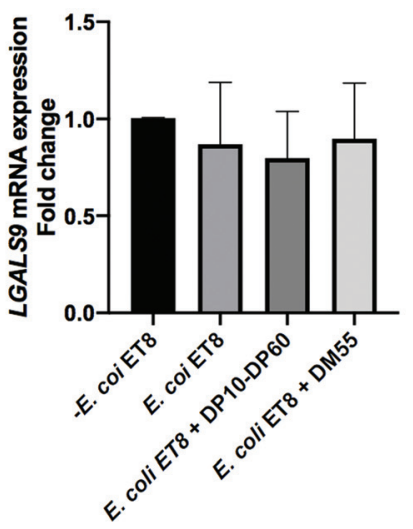

F

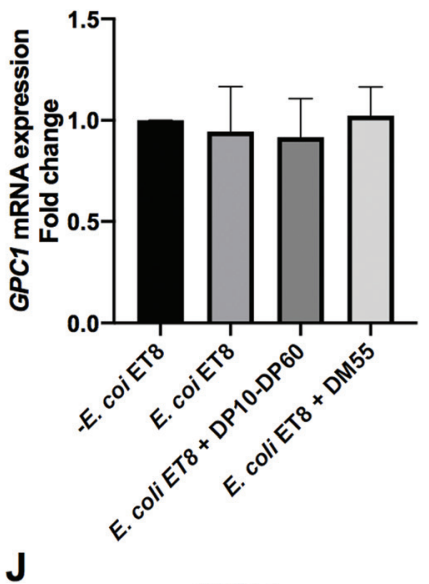

CXCL3

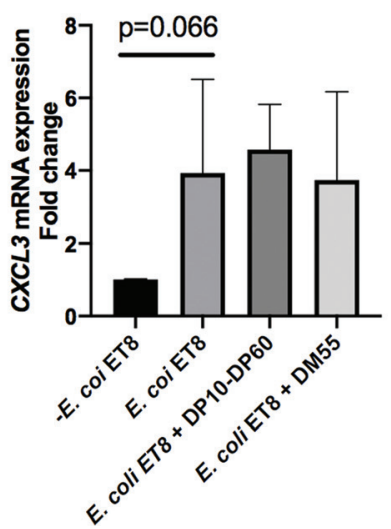

C

HAS3

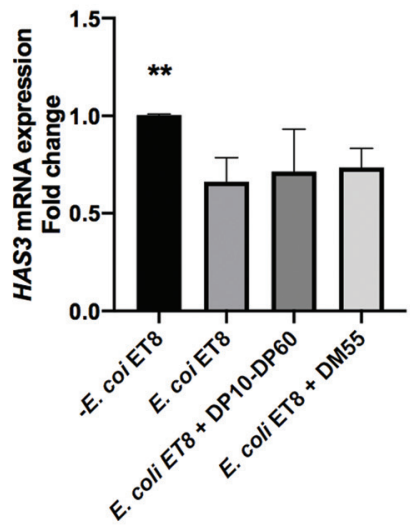

G

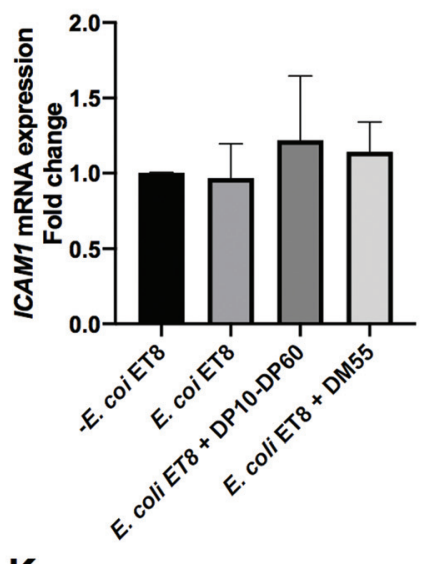

CCL20

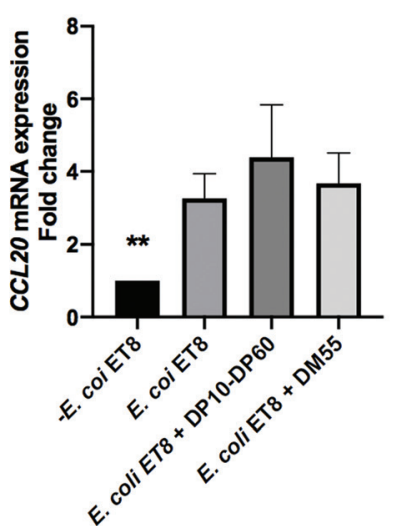

D EXT1

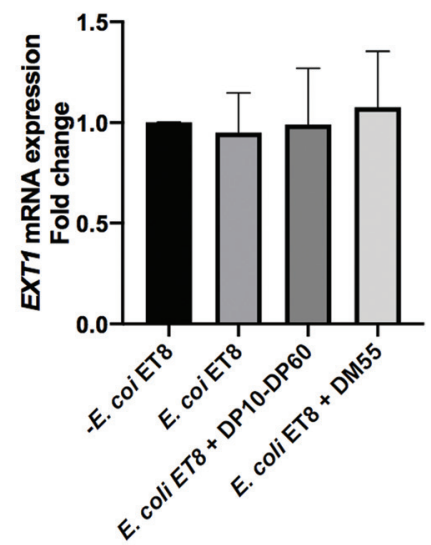

H

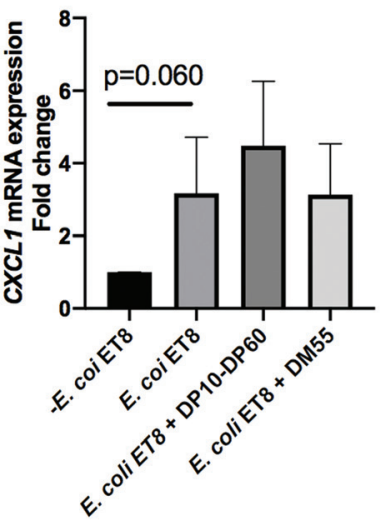

$\mathbf{L}$

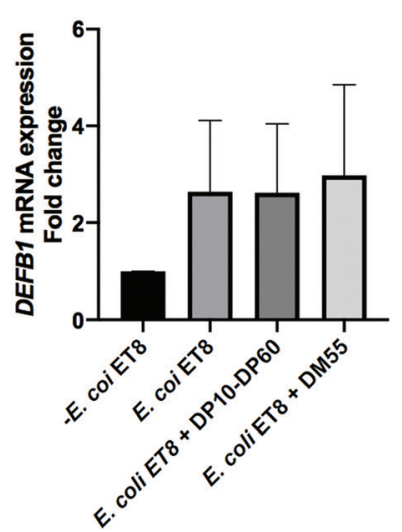

Fig. 4 E. coli ET8 infection and the effects of DP10-DP60 inulin and DM55 pectin on cell membrane glycosylation and inflammation genes in the intestinal epithelial Caco-2 cells. Caco-2 cells were cultured for 21 days and pre-incubated with DP10-DP60 inulin and DM55 pectin for $2 \mathrm{~h}$ before infection with E. coli ET8 harvested from the log phase. After infection, the cell membrane glycosylation genes [galectins (LGALS1 and LGALS9) and glycocalyx (HAS3, EXT1, EXT2 and GPC1)] and inflammation associated genes [cell adhesion and chemotaxis target genes (ICAM1, CXCL1, CXCL2, $C X C L 3$ and $C C L 20$ ) and the anti-microbial peptide gene (DEFB1)] were quantified by evaluating the mRNA expression with real-time RT-PCR. The results are presented as a fold change compared to the group without E. coli ET8 infection. Statistical analysis was performed using one-way ANOVA, comparing to the group only with $E$. coli ET8 infection, the group without $E$. coli ET8 infection and the group with pre-incubation of carbohydrates prior to $E$. coli ET8 infection $\left({ }^{*} p<0.05,{ }^{* *} p<0.01\right.$ and $p<0.1$ were defined as a statistical trend). 
A

LGALS1

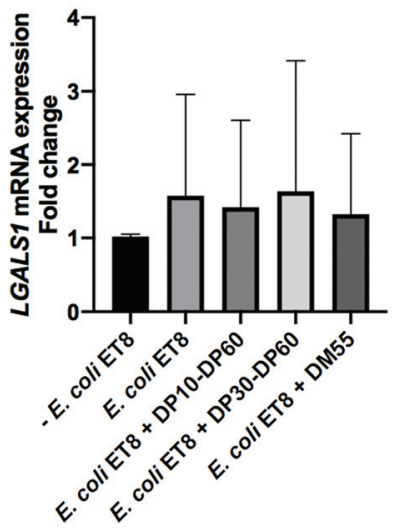

E

EXT2
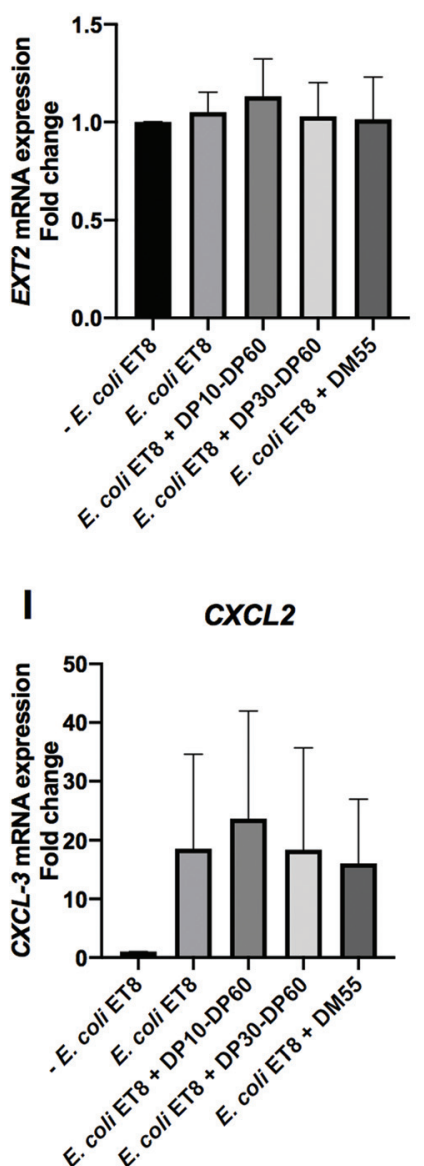

B

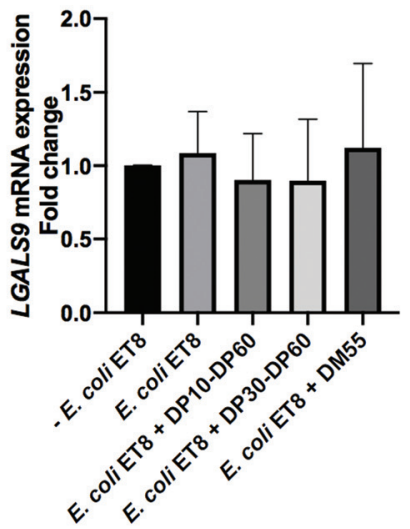

$\mathbf{F}$

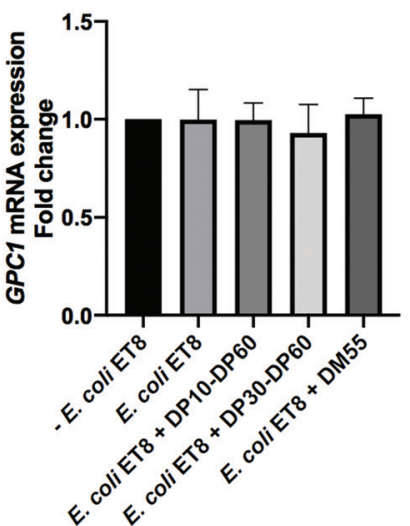

$\mathbf{J}$

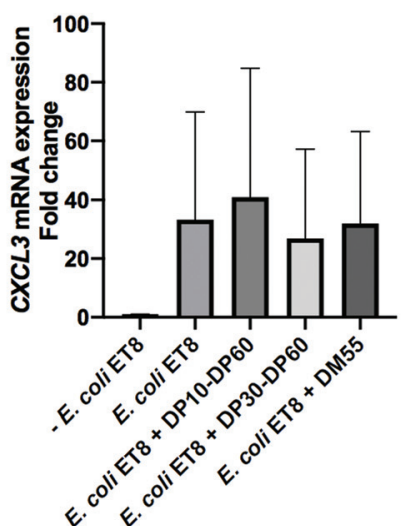

C

HAS3

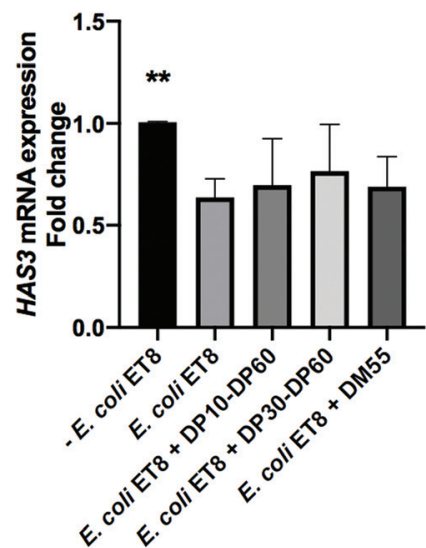

G ICAM1
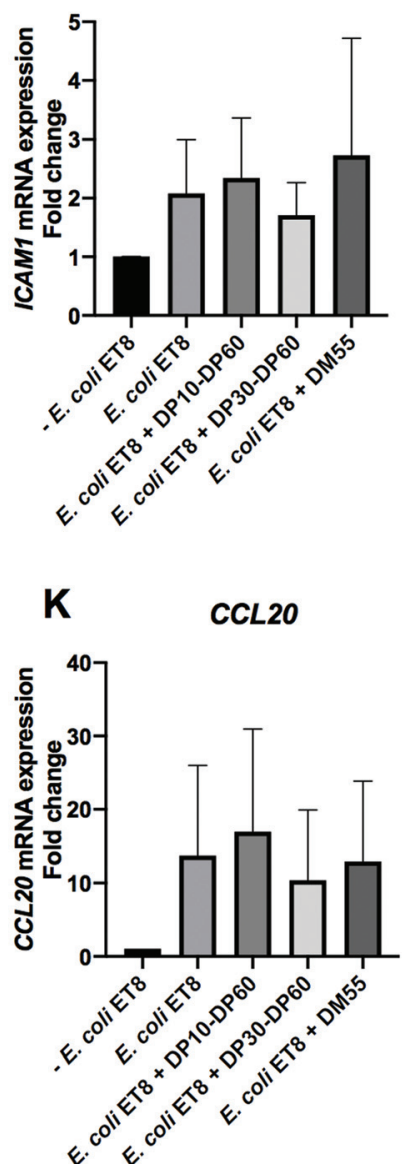

D EXT1

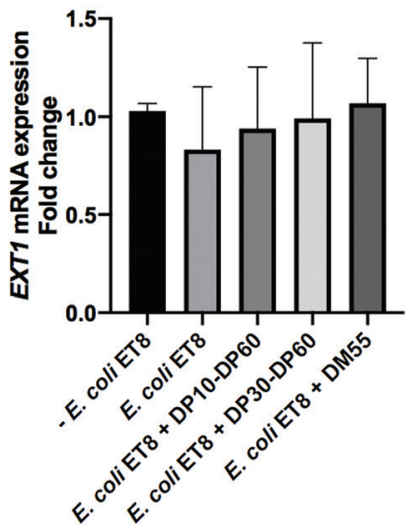

H CXCL1

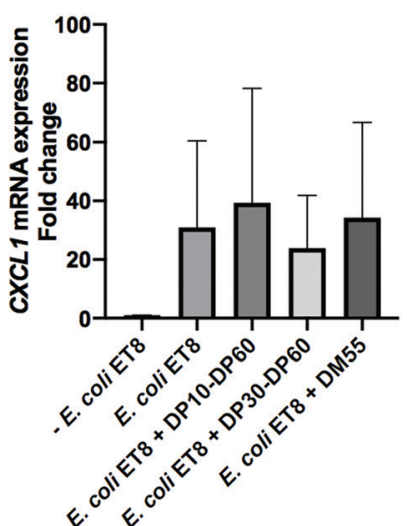

L

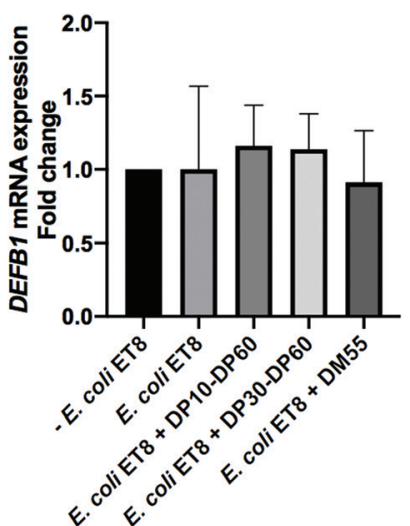

Fig. 5 E. coli ET8 infection and the effects of DP10-DP60 and DP30-DP60 inulins and DM55 pectin on the cell membrane glycosylation and inflammation genes in intestinal epithelial Caco-2 cells. Caco-2 cells were cultured for 21 days and pre-incubated with DP10-DP60 and DP30DP60 inulins and DM55 pectin for $2 \mathrm{~h}$ before infection with E. coli ET8 harvested from the stationary phase. After infection, the cell membrane glycosylation genes [galectins (LGALS1 and LGALS9) and glycocalyx (HAS3, EXT1, EXT2 and GPC1)] and inflammation associated genes [cell adhesion and chemotaxis target genes (ICAM1, CXCL1, CXCL2, CXCL3 and CCL20) as well as the anti-microbial peptide gene (DEFB1)] were quantified by evaluating the mRNA expression with real-time RT-PCR. The results are presented as a fold change compared to the group without $E$. coli $\mathrm{ET} 8$ infection. Statistical analysis was performed using one-way ANOVA, comparing to the group only with E. coli ET8 infection, the group without E. coli ET8 infection and the group with pre-incubation of carbohydrates prior to E. coli ET8 infection (** $p<0.01)$. 
A

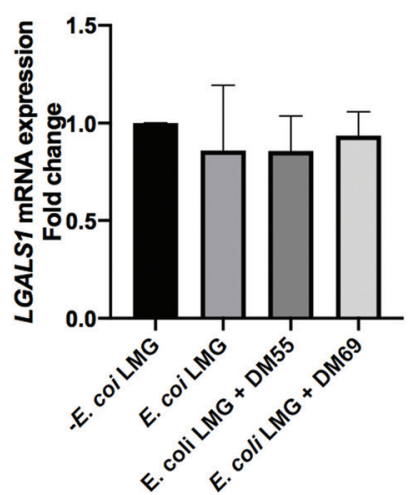

E

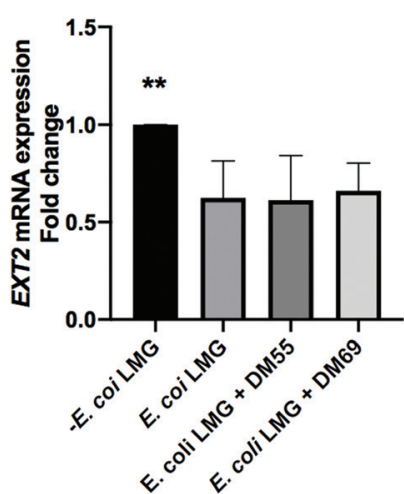

I

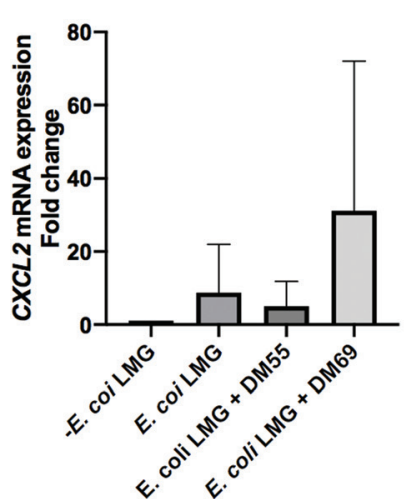

B

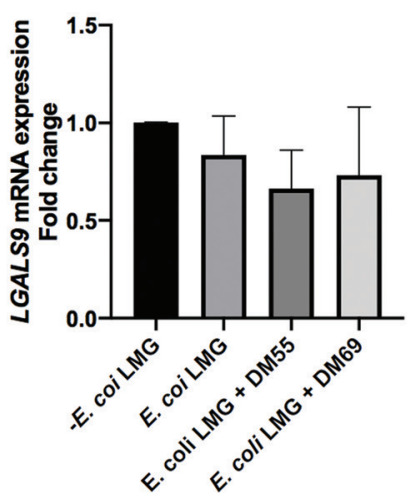

$\mathbf{F}$

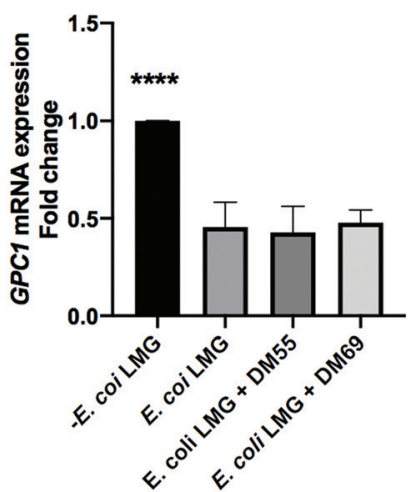

J

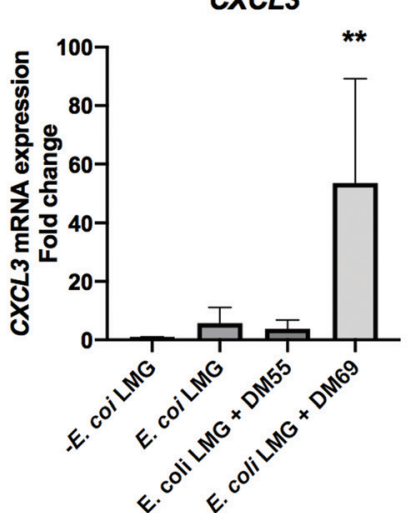

C

HAS3

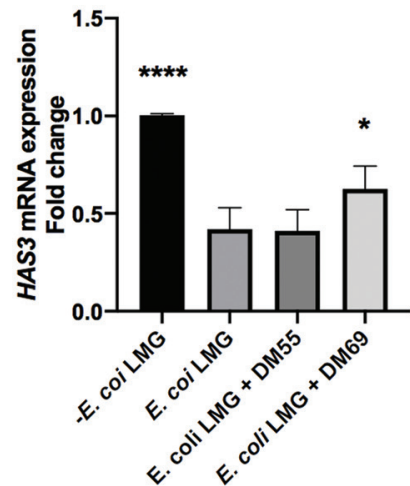

G

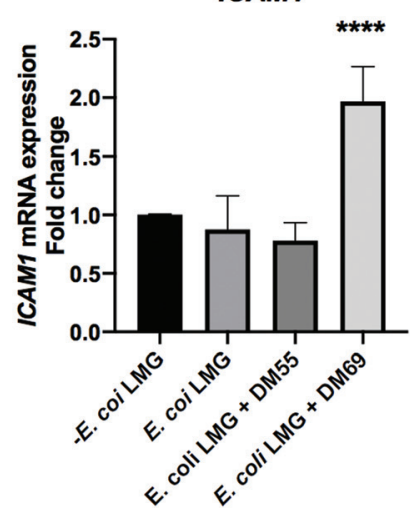

K

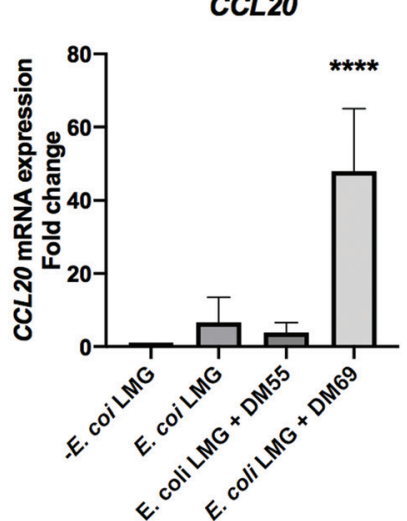

D

EXT1

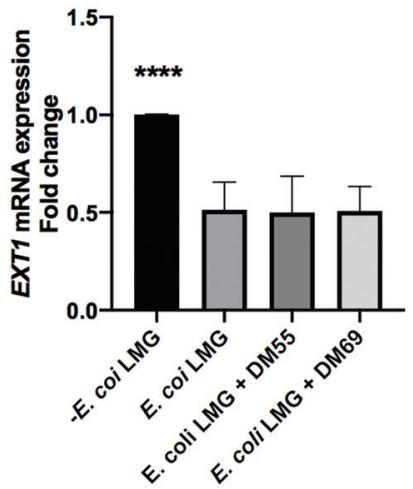

H

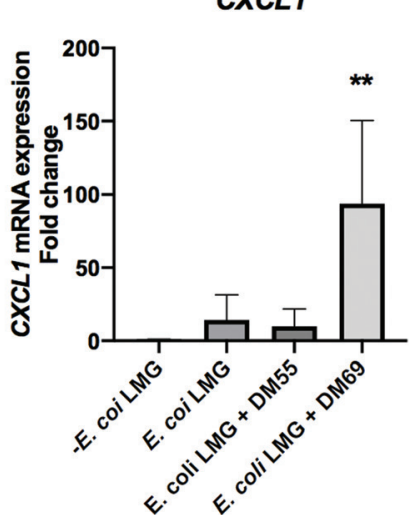

$\mathbf{L}$

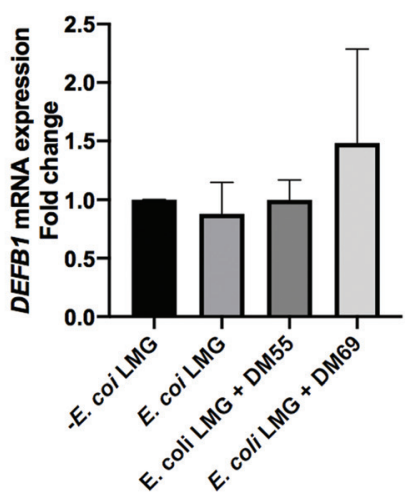

Fig. 6 E. coli LMG5862 infection and the effects of DM55 and DM69 pectins on the cell membrane glycosylation and inflammation genes in intestinal epithelial Caco-2 cells. Caco-2 cells were cultured for 21 days and pre-incubated with DM55 and DM69 pectins for $2 \mathrm{~h}$ before infection with E. coli LMG5862 harvested from the log phase. After infection, the cell membrane glycosylation genes [galectins (LGALS1 and LGALS9) and glycocalyx (HAS3, EXT1, EXT2 and GPC1)] and inflammation associated genes [cell adhesion and chemotaxis target genes (ICAM1, CXCL1, CXCL2, CXCL3 and $C C L 20$ ) as well as the anti-microbial peptide gene (DEFB1)] were quantified by evaluating the mRNA expression with real-time RT-PCR. The results are presented as a fold change compared to the group without E. coli LMG5862 infection. Statistical analysis was performed using one-way ANOVA, comparing to the group only with E. coli LMG5862 infection, the group without $E$. coli LMG5862 infection and the group with pre-incubation of carbohydrates prior to $E$. coli LMG5862 infection $\left({ }^{*} p<0.05,{ }^{* *} p<0.01\right.$ and $\left.{ }^{* * * *} p<0.0001\right)$. 
A

LGALS1

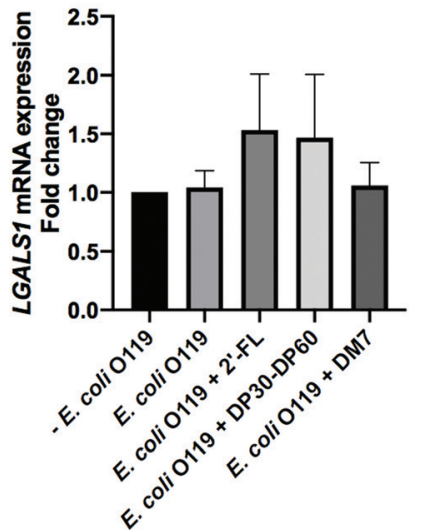

E

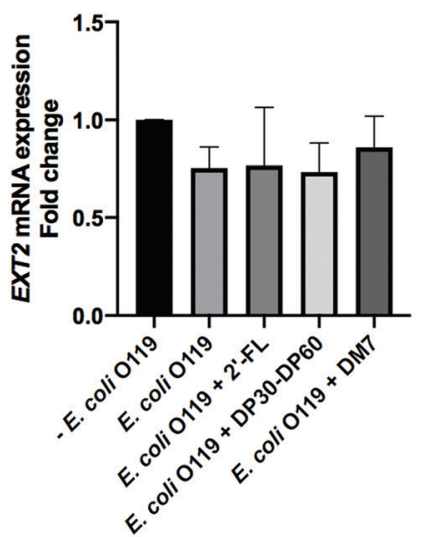

I

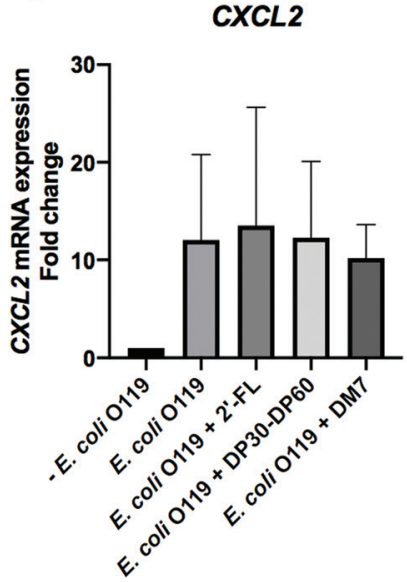

B

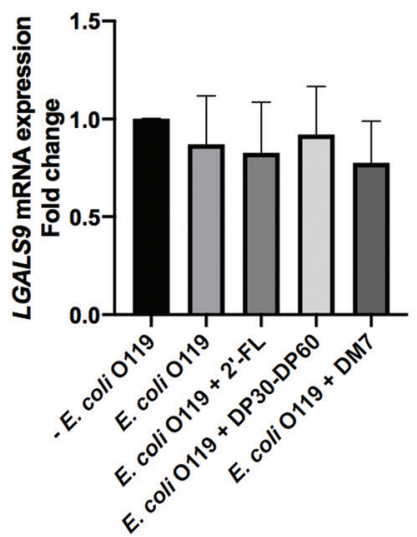

$\mathbf{F}$

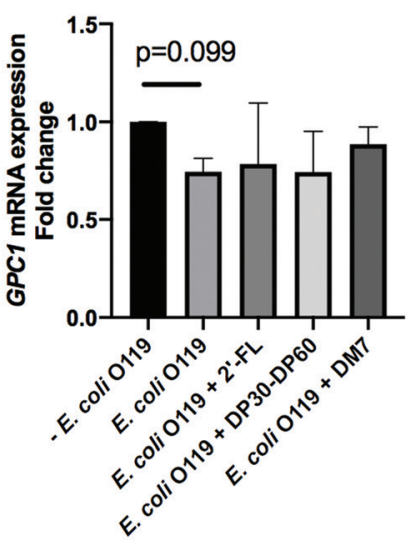

CXCL3

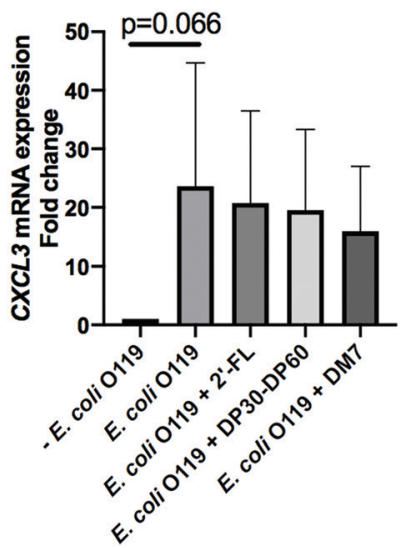

C

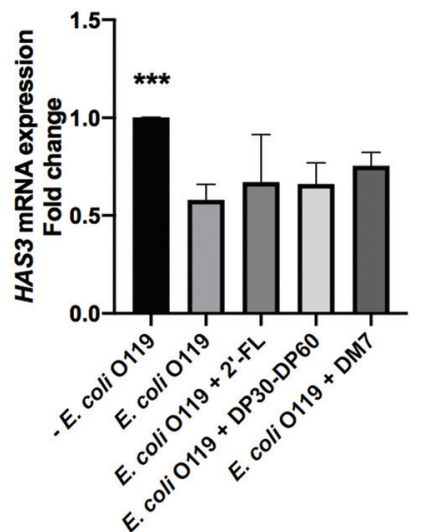

G

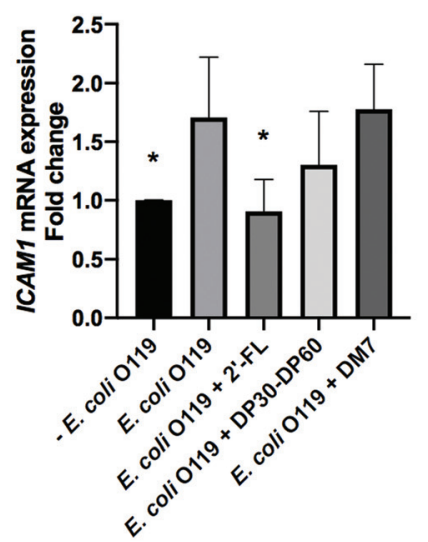

K

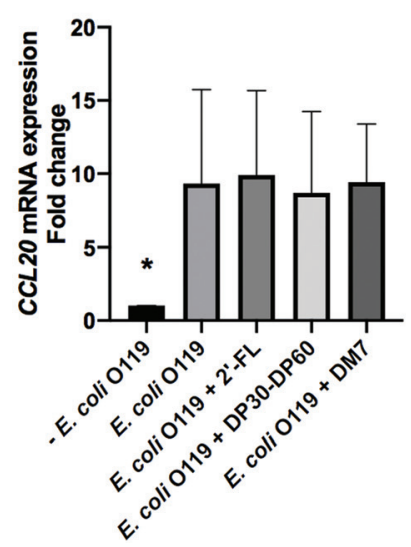

EXT1

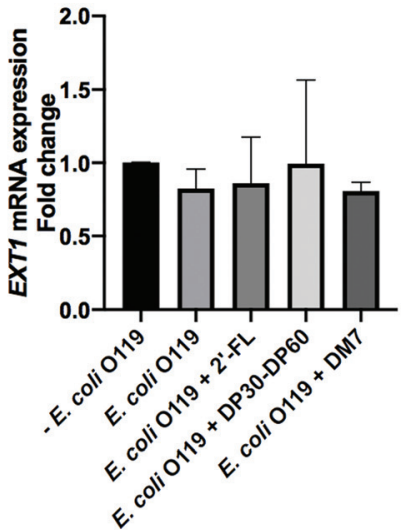

H

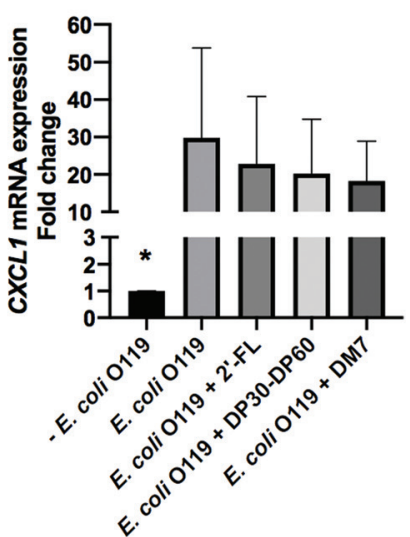

$\mathbf{L}$

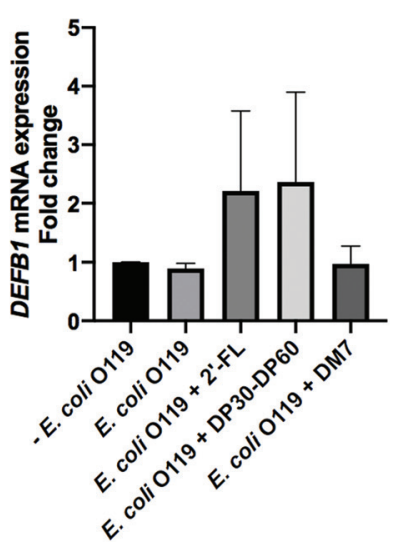

Fig. 7 E. coli 0119 infection and the effects of 2'-FL, DP30-DP60 inulin, and DM7 pectin on the cell membrane glycosylation and inflammation genes in intestinal epithelial Caco-2 cells. Caco-2 cells were cultured for 21 days and pre-incubated with 2'-FL, DP30-DP60 inulin, and DM7 pectin for $2 \mathrm{~h}$ before infection with E. coli O119 harvested from the log phase. After infection, the cell membrane glycosylation genes [galectins (LGALS1 and LGALS9) and glycocalyx (HAS3, EXT1, EXT2 and GPC1)] and inflammation associated genes [cell adhesion and chemotaxis target genes (ICAM1, CXCL1, CXCL2, CXCL3 and CCL20) as well as the anti-microbial peptide gene (DEFB1)] were quantified by evaluating the mRNA expression with real-time RT-PCR. The results are presented as a fold change compared to the group without $E$. coli O119 infection. Statistical analysis was performed using one-way ANOVA, comparing to the group only with E. coli O119 infection, the group without $E$. coli O119 infection and the group with pre-incubation of carbohydrates prior to $E$. coli 0119 infection $\left({ }^{*} p<0.05,{ }^{* \star} p<0.001\right.$ and $p<0.1$ were considered a statistical trend). 
A

LGALS1

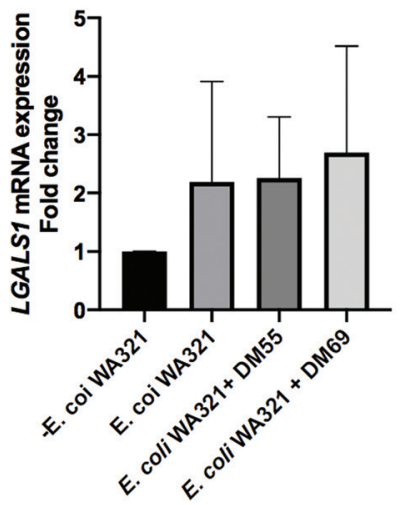

E

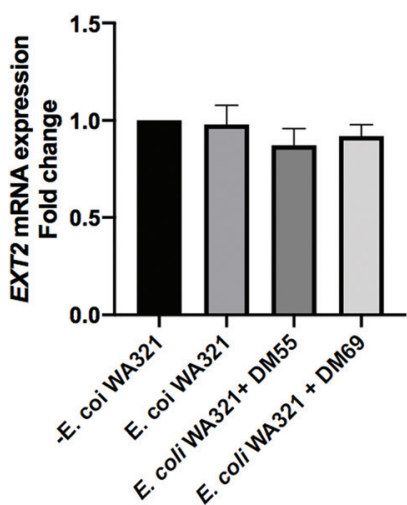

I

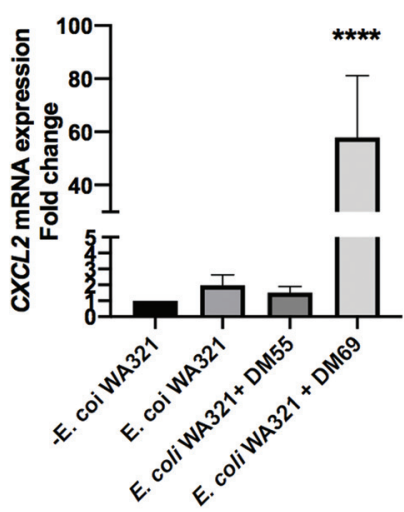

B

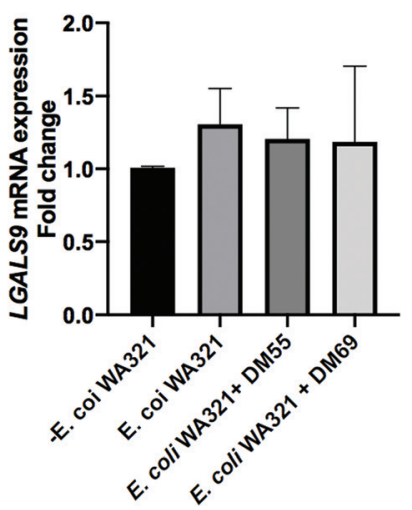

F

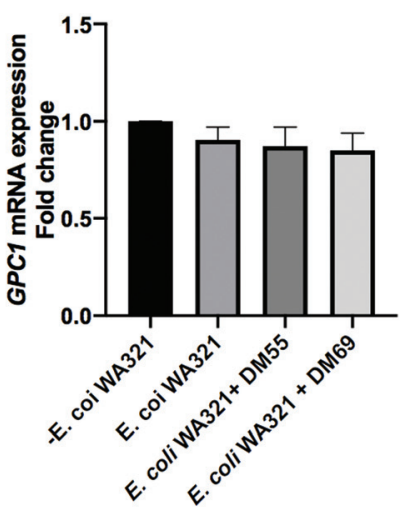

J

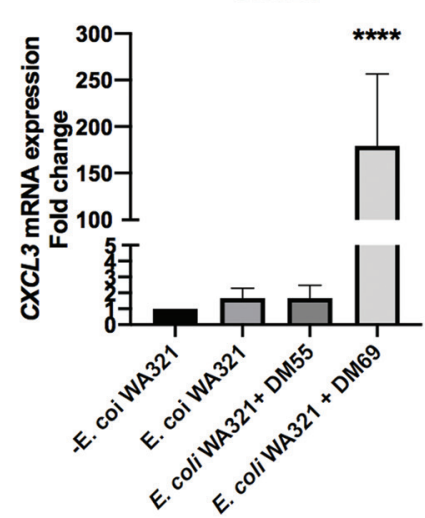

C

HAS3

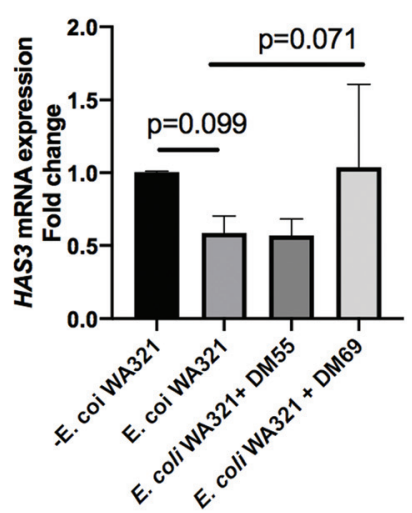

G

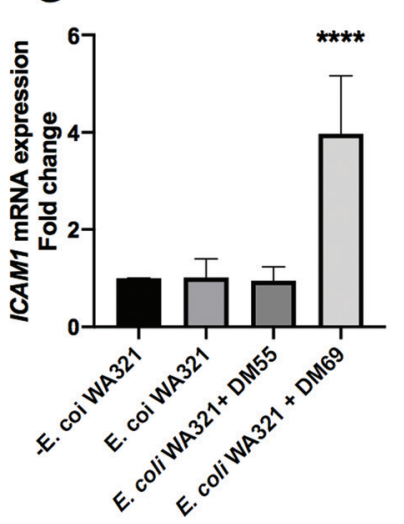

K

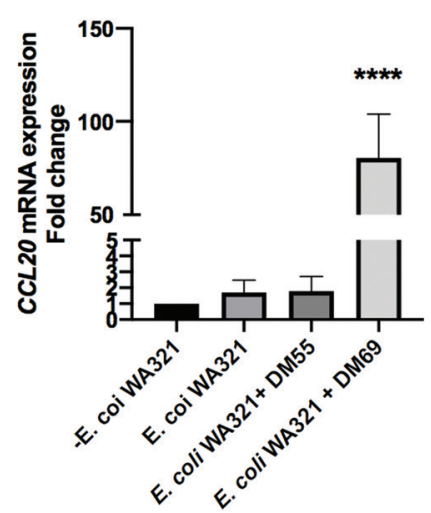

D

EXT1

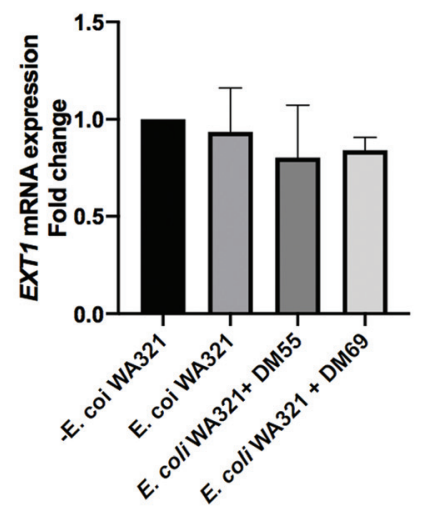

H

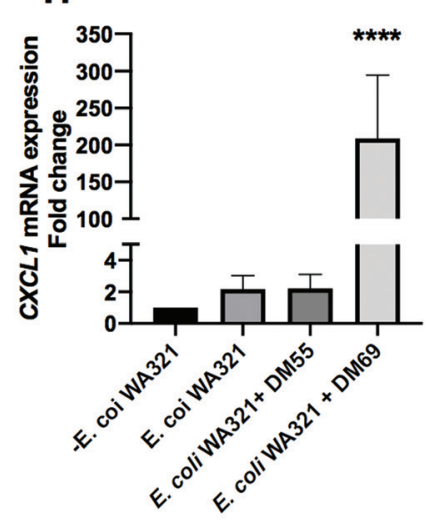

$\mathbf{L}$

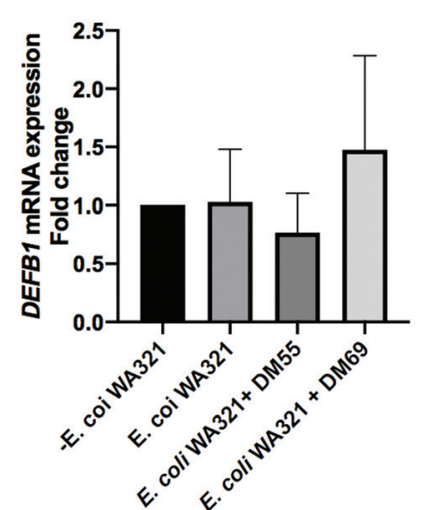

Fig. 8 E. coli WA321 infection and the effects of DM55 and DM69 pectins on the cell membrane glycosylation and inflammation genes in intestinal epithelial Caco-2 cells. Caco-2 cells were cultured for 21 days and pre-incubated with DM55 and DM69 pectins for $2 \mathrm{~h}$ before infection with E. coli WA321 harvested from the stationary phase. After infection, the cell membrane glycosylation genes [galectins (LGALS1 and LGALS9) and glycocalyx (HAS3, EXT1, EXT2 and GPC1)] and inflammation associated genes [cell adhesion and chemotaxis target genes (ICAM1, CXCL1, CXCL2, CXCL3 and $C C L 20)$ as well as the anti-microbial peptide gene (DEFB1)] were quantified by evaluating the mRNA expression with real-time RT-PCR. The results are presented as a fold change compared to the group without E. coli WA321 infection. Statistical analysis was performed using one-way ANOVA, comparing to the group only with $E$. coli WA321 infection, the group without $E$. coli WA321 infection and the group with pre-incubation of carbohydrates prior to $E$. coli WA321 infection ( ${ }^{* \star * *} p<0.0001$ and $p<0.1$ were considered a statistical trend). 
A

LGALS1

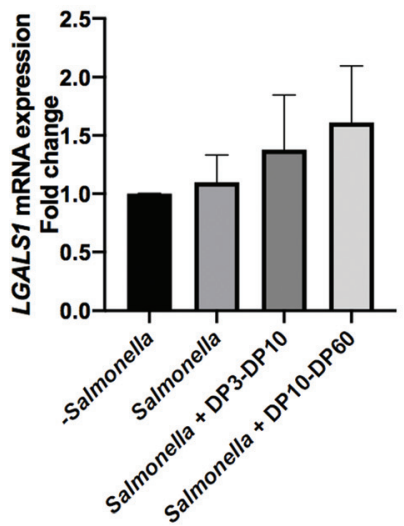

E

EXT2

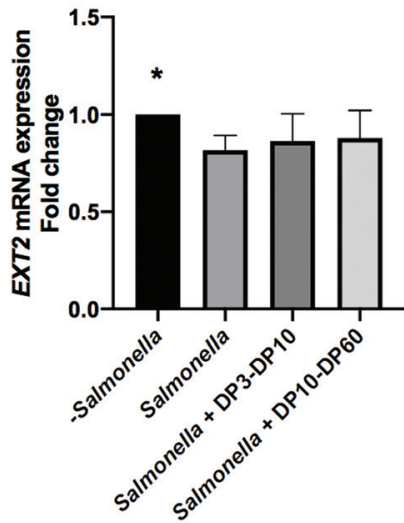

CXCL2

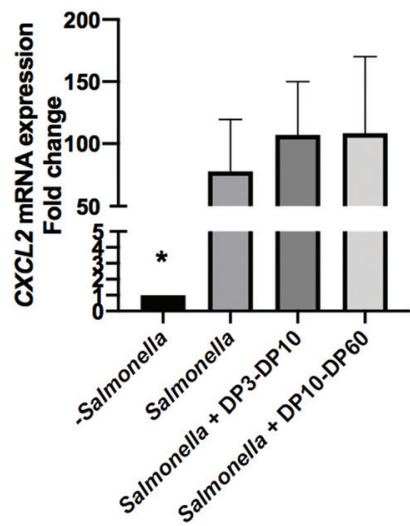

B

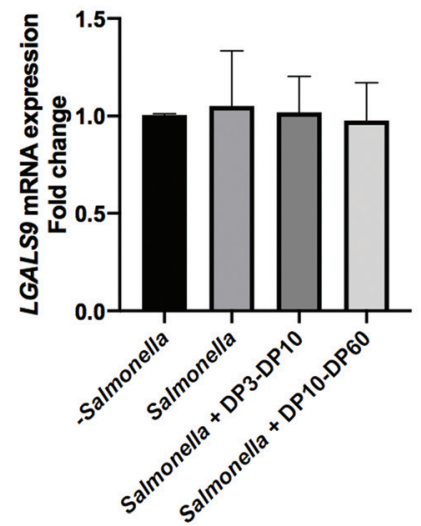

F

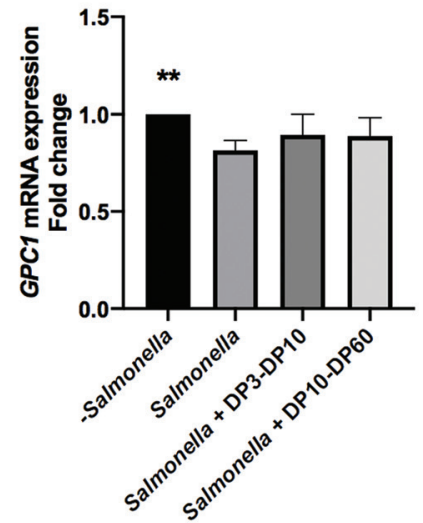

CXCL3

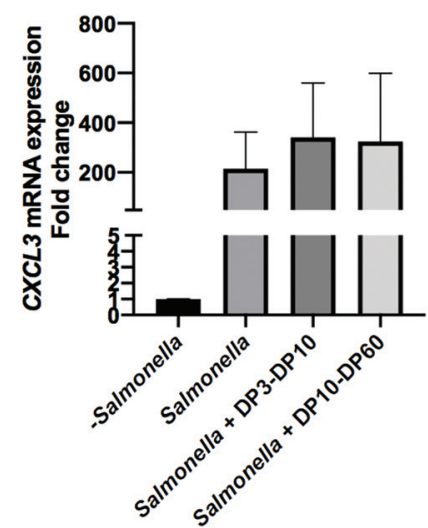

C

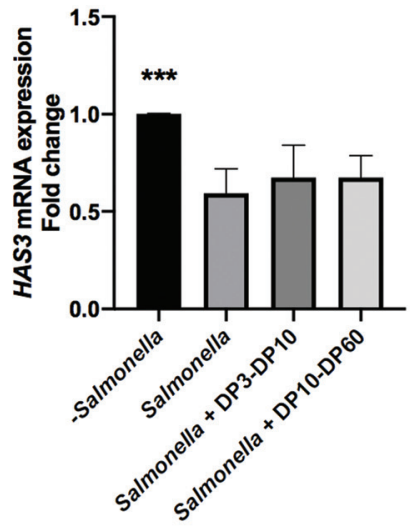

G

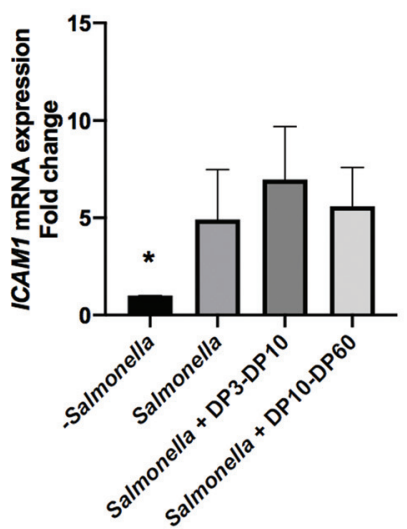

K

CCL20

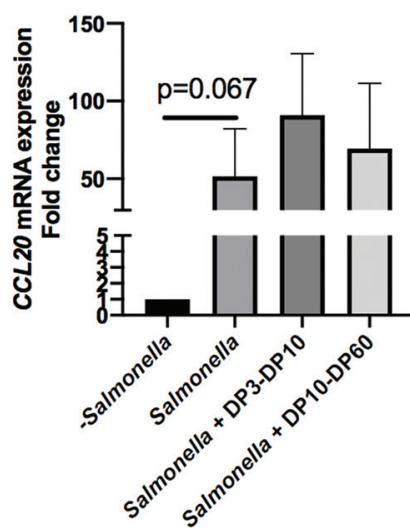

EXT1

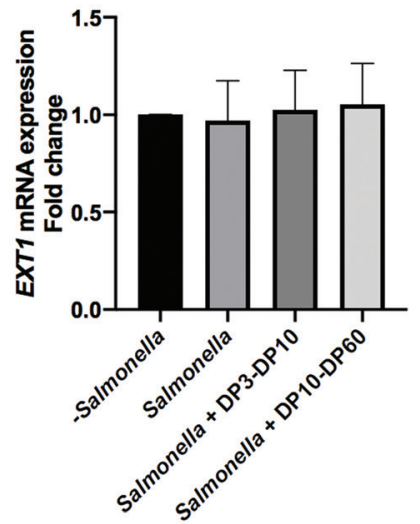

H

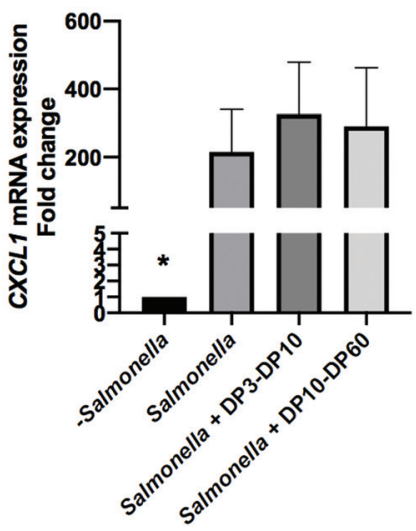

$\mathbf{L}$

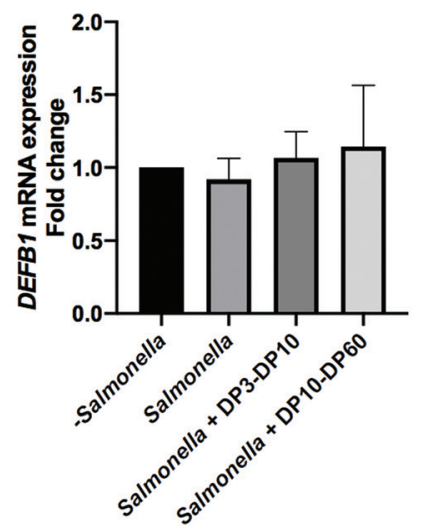

Fig. 9 S. enterica subsp. enterica LMG07233 infection and the effects of DP3-DP10 and DP10-DP60 pectins on the cell membrane glycosylation and inflammation genes in intestinal epithelial Caco-2 cells. Caco-2 cells were cultured for 21 days and pre-incubated with DP3-DP10 and DP10DP60 inulins for $2 \mathrm{~h}$ before infection with S. enterica subsp. enterica LMG07233 harvested from the stationary phase. After infection, the cell membrane glycosylation genes [galectins (LGALS1 and LGALS9) and glycocalyx (HAS3, EXT1, EXT2 and GPC1)] and inflammation associated genes [cell adhesion and chemotaxis target genes (ICAM1, CXCL1, CXCL2, CXCL3 and CCL20) as well as the anti-microbial peptide gene (DEFB1)] were quantified by evaluating the mRNA expression with real-time RT-PCR. The results are presented as a fold change compared to the group without S. enterica subsp. enterica LMG07233 infection. Statistical analysis was performed using one-way ANOVA, comparing to the group only with S. enterica subsp. enterica LMG07233 infection, the group without S. enterica subsp. enterica LMG07233 infection and the group with pre-incubation of carbohydrates prior to $S$. enterica subsp. enterica LMG07233 infection $\left({ }^{*} p<0.05,{ }^{* *} p<0.01\right.$, ${ }^{* *} p<0.001$ and $p<0.1$ were considered a statistical trend). 
3.97-fold (Fig. 8G, $p<0.0001$ ), 208.97-fold (Fig. 8H, $p<0.0001$ ), 57.88-fold (Fig. 8I, $p<0.0001$ ), 179.11-fold (Fig. 8J, $p<0.0001$ ), and 78.66-fold (Fig. 8K, $p<0.0001$ ), respectively. This upregulatory effect of DM69 pectin was not achieved by DM55 pectin.

3.4.5. S. enterica subsp. enterica LMG07233. Stationary phase. S. enterica subsp. enterica LMG07233 from the stationary phase significantly downregulated the glycosylation genes including HAS3, EXT2, and GPC1 in Caco-2 cells, with a reduction of 40.89\% (Fig. 9C, $p<0.001$ ), 18.31\% (Fig. 9E, $p<0.05$ ), and $18.59 \%$ (Fig. 9F, $p<0.01$ ), respectively. The adhesion and chemotaxis target genes ICAM1, CXCL1, and CXCL2 were significantly upregulated, with an increase of 3.92-fold (Fig. 9G, $p<0.05$ ), 214.26-fold (Fig. 9H, $p<0.05$ ), and 76.73-fold (Fig. 9I, $p<0.05$ ), respectively. S. enterica subsp. enterica LMG07233 showed a tendency to upregulate CCL2O by 50.53 -fold (Fig. 9K, $p=0.067$ ). DEFB1 was not influenced by the infection of $S$. enterica subsp. enterica LMG07233. DP3-DP10 and DP10-DP60 inulins had no effect on the above genes.

3.4.6. K. pneumoniae LMG20218. Log phase. Consistently, K. pneumoniae LMG20218 from the log phase significantly downregulated the glycosylation genes $H A S 3, E X T 1$, and GPC1, and reduced it by $54.32 \%$ (Fig. 10C, $p<0.0001$ ), $37.25 \%$ (Fig. 10D, $p<0.0001$ ), and $20.54 \%$ (Fig. 10F, $p<0.05$ ), respectively. Among the adhesion and chemotaxis target genes, only ICAM1 was significantly downregulated by $33.51 \%$ (Fig. 10G, $p$ $<$ 0.01). DEFB1 was not influenced by $K$. pneumoniae LMG20218. Pre-incubating Caco-2 cells with DM7 pectin significantly upregulated HAS3 and EXT1 by $9.62 \%$ (Fig. 10C, $p<$ 0.05 ) and $12.68 \%$ (Fig. 10D, $p<0.05$ ), respectively. ICAM1 was significantly upregulated after pre-incubating Caco-2 cells with DM7 and DM55 pectins, with an increase of 38.29\% (Fig. 10G, $p<0.001$ ) and 25.26\% (Fig. 10G, $p<0.05$ ), respectively.

Stationary phase. K. pneumoniae LMG20218 from the stationary phase significantly downregulated HAS3 by $40.15 \%$ (Fig. $11 \mathrm{C}, p<0.0001$ ). Pre-incubating Caco-2 cells with carbohydrates resulted in pronounced effects as observed after K. pneumoniae LMG20218 exposure. The glycosylation genes of LGALS1 and HAS3 were significantly upregulated by DM69 pectin with an increase of $34.52 \%$ (Fig. 11A, $p<0.05$ ) and 15.69\% (Fig. 11C, $p<0.05$ ), respectively. However, DM55 pectin significantly downregulated LGALS9 and GPC1 by 21.84\% (Fig. 11B, $p<0.05$ ) and 16.75\% (Fig. 11F, $p<0.05$ ), respectively. DM7 pectin also significantly downregulated GPC1 by $14.62 \%$ (Fig. $11 \mathrm{~F}, p<0.05$ ). The adhesion and chemotaxis target genes ICAM1, CXCL1, CXCL2, CXCL3, and CCL2O were all significantly upregulated by DM69 pectin, with an increase of 2.35-fold (Fig. 11G, $p<0.0001$ ), 77.49-fold (Fig. 11H, $p<0.0001$ ), 27.51-fold (Fig. 11I, $p<0.01$ ), 47.03-fold (Fig. 11J, $p<0.0001$ ), and 67.19-fold (Fig. 11K, $p<0.0001$ ), respectively. DM69 pectin pre-incubation also significantly upregulated DEFB1 by $79.45 \%$ (Fig. 11L, $p<0.01$ ).

\section{Discussion}

Previous studies have shown that hMOs and NDCs can modulate the gut epithelial cells and thus result in lower chances for pathogen infection. ${ }^{40,41}$ It is however largely unknown which mechanisms are responsible for the anti-adhesion effects of hMOs and NDCs and whether these are pathogen specific. This knowledge is of significant importance for the effective tailoring of infant formula to modulate gut epithelial cells to resist pathogen infections. This was one of the reasons to perform this study and we show that specific structures of hMOs, inulins and pectins induce anti-adhesion effects of specific pathogens from different growth phases. We show that these specific hMO and NDC structures modulate intestinal epithelial glycosylation genes and inflammation associated genes, which may explain the anti-adhesion effects of the hMO and NDC structures on these pathogens towards gut epithelial cells.

Our data show that pre-incubating Caco-2 cells with the hMO 2'-FL but not with 3-FL inhibit the adhesion of $E$. coli O119 from the log phase. The specific pathogen adhesion inhibitory effect of 2 '-FL might be explained by the downregulation of the gene expression of intercellular adhesion molecule-1 (ICAM1) on Caco-2 cells. ICAM1 is a trans-membrane receptor that is expressed in the intestine and involved in the inflammatory process induced by pathogens. ${ }^{18}$ This may indicate that 2'-FL can directly influence the expression of the intestinal epithelial receptor ICAM1, which is probably essential for the adhesion of $E$. coli O119. Although both 2'-FL and 3-FL belong to the fucosylated hMO and have the same lactose core structure, it is probably the difference in fucosylation that should explain the difference in their biological effects. ${ }^{42}$

All the inulins tested in this study showed an inhibitory effect on the adhesion of pathogens to Caco- 2 cells, but the magnitude of prevention of adhesion was shown to be dependent on the chain length of the inulins. The short chain inulin DP3-DP10 only inhibited the adhesion of $S$. enterica subsp. enterica LMG07233 from the stationary phase, while the long chain inulin DP10-DP60 and DP30-DP60 inulin induced a larger inhibitory effect on pathogen adhesion. The DP10-DP60 inulin inhibited the adhesion of E. coli ET8 from both log and stationary phases, and S. enterica subsp. enterica LMG07233 from the stationary phase. The DP30-DP60 inulin inhibited the gut pathogens of E. coli 0119 from the log phase, E. coli ET8 from the stationary phase and one lung pathogen of K. pneumoniae LMG20218 from the stationary phase. The antiadhesion effects by inulins were neither induced by the cell membrane glycosylation nor by the expressions of the tested inflammation associated genes. Other genes such as mucosal immunity and barrier function related genes might be involved in the anti-adhesion effects of inulins on the pathogens. ${ }^{43}$ Effects of different inulins were also dependent on the growth phase of the pathogens. Pathogens in different growth phases contribute differently to the infection process. ${ }^{44}$ For instance, E. coli was demonstrated to be more adhesive and infectious in the stationary phase than in the log phase. ${ }^{44}$ Our data show that pre-incubating Caco- 2 cells with especially the longer chain DP30-DP60 inulin inhibited the adhesion of E. coli ET8 from the stationary phase but not from the log phase, illustrating that inulins may have different efficacies on 
A

LGALS1

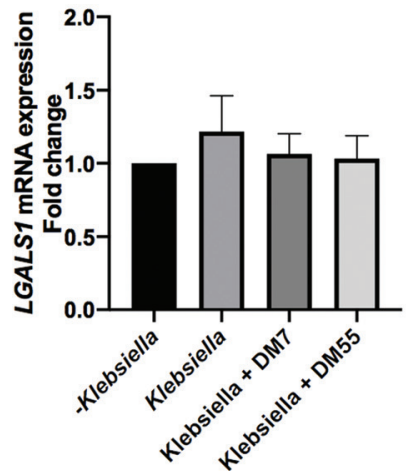

E

EXT2

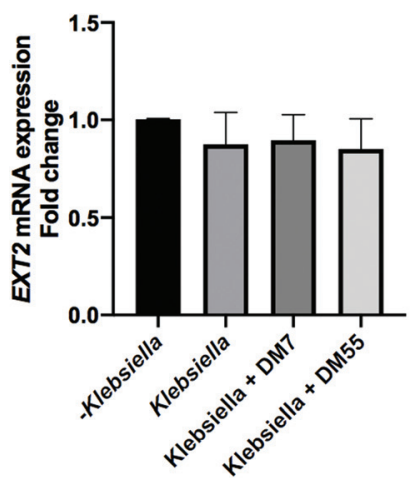

I

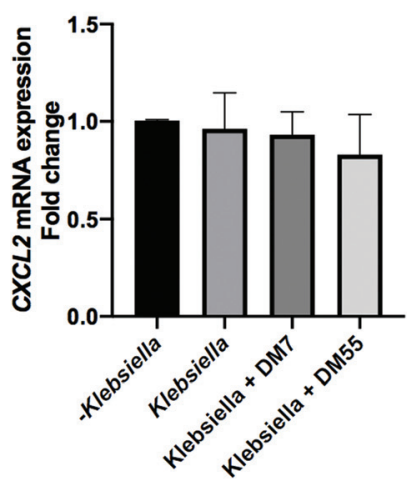

B

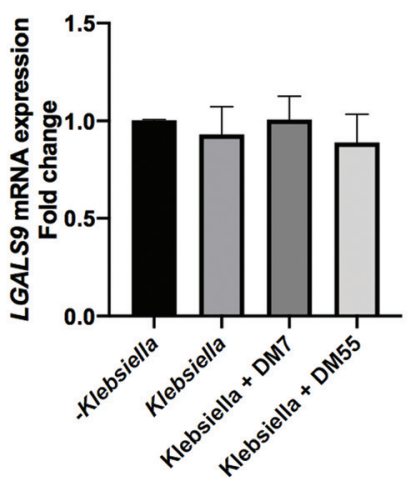

F

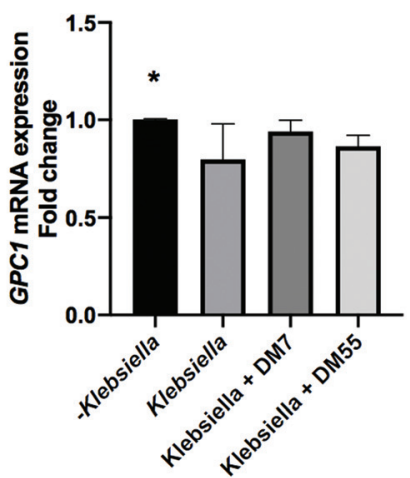

J

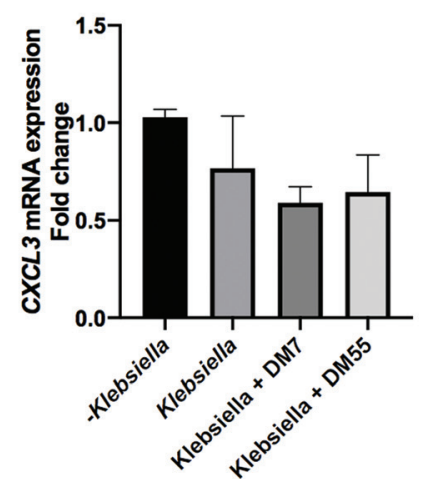

C

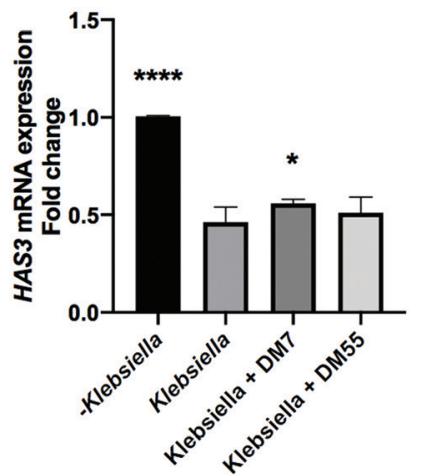

G

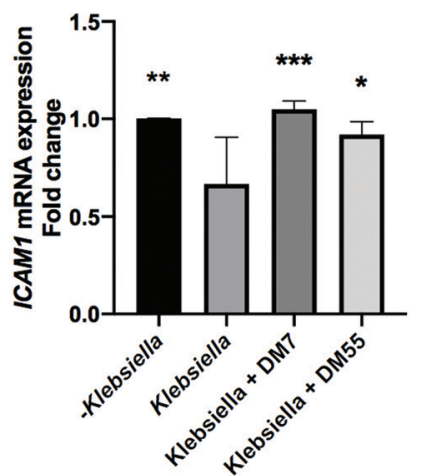

K

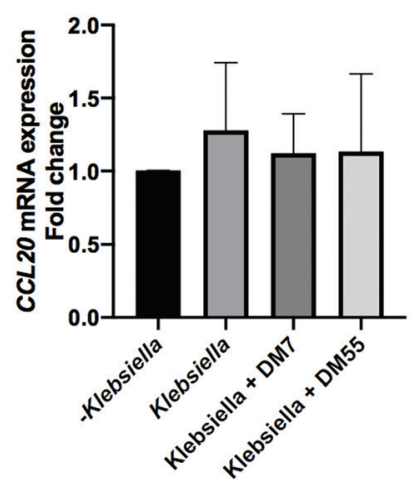

D

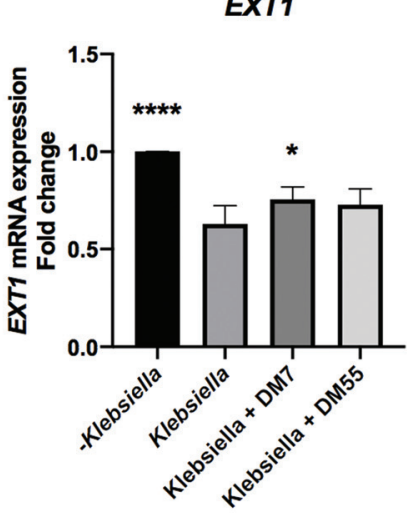

H

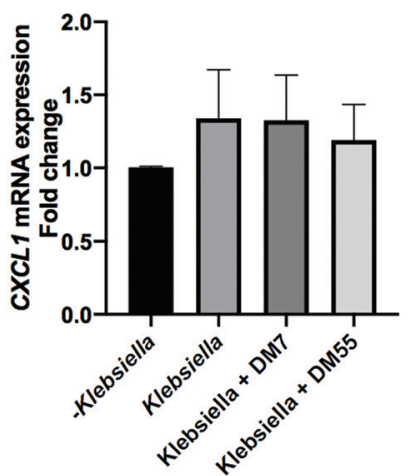

L

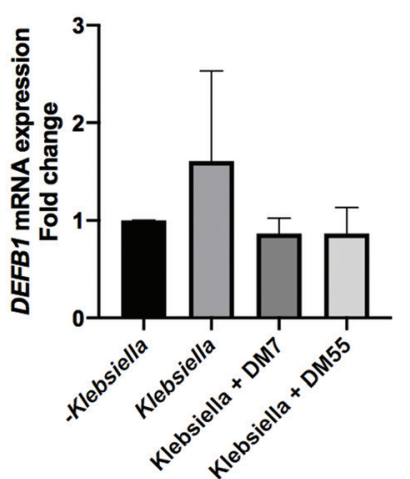

Fig. 10 K. pneumoniae LMG20218 infection and the effects of DM7 and DM55 pectins on the cell membrane glycosylation and inflammation genes in intestinal epithelial Caco-2 cells. Caco-2 cells were cultured for 21 days and pre-incubated with DM7 and DM55 pectins for $2 \mathrm{~h}$ before infection with $K$. pneumoniae LMG20218 harvested from the log phase. After infection, the cell membrane glycosylation genes [galectins (LGALS1 and LGALS9) and glycocalyx (HAS3, EXT1, EXT2 and GPC1)] and inflammation associated genes [cell adhesion and chemotaxis target genes (ICAM1, CXCL1, CXCL2, CXCL3 and CCL20) as well as the anti-microbial peptide gene (DEFB1)] were quantified by evaluating the mRNA expression with real-time RT-PCR. The results were presented as a fold change compared to the group without $K$. pneumoniae LMG20218 infection. Statistical analysis was performed using one-way ANOVA, comparing to the group only with $K$. pneumoniae LMG20218 infection, the group without $K$. pneumoniae LMG20218 infection and the group with pre-incubation of carbohydrates prior to $K$. pneumoniae LMG20218 infection $(* p<0.05, * * p$ $<0.01,{ }^{* * *} p<0.001$ and $\left.{ }^{* * * *} p<0.0001\right)$. 

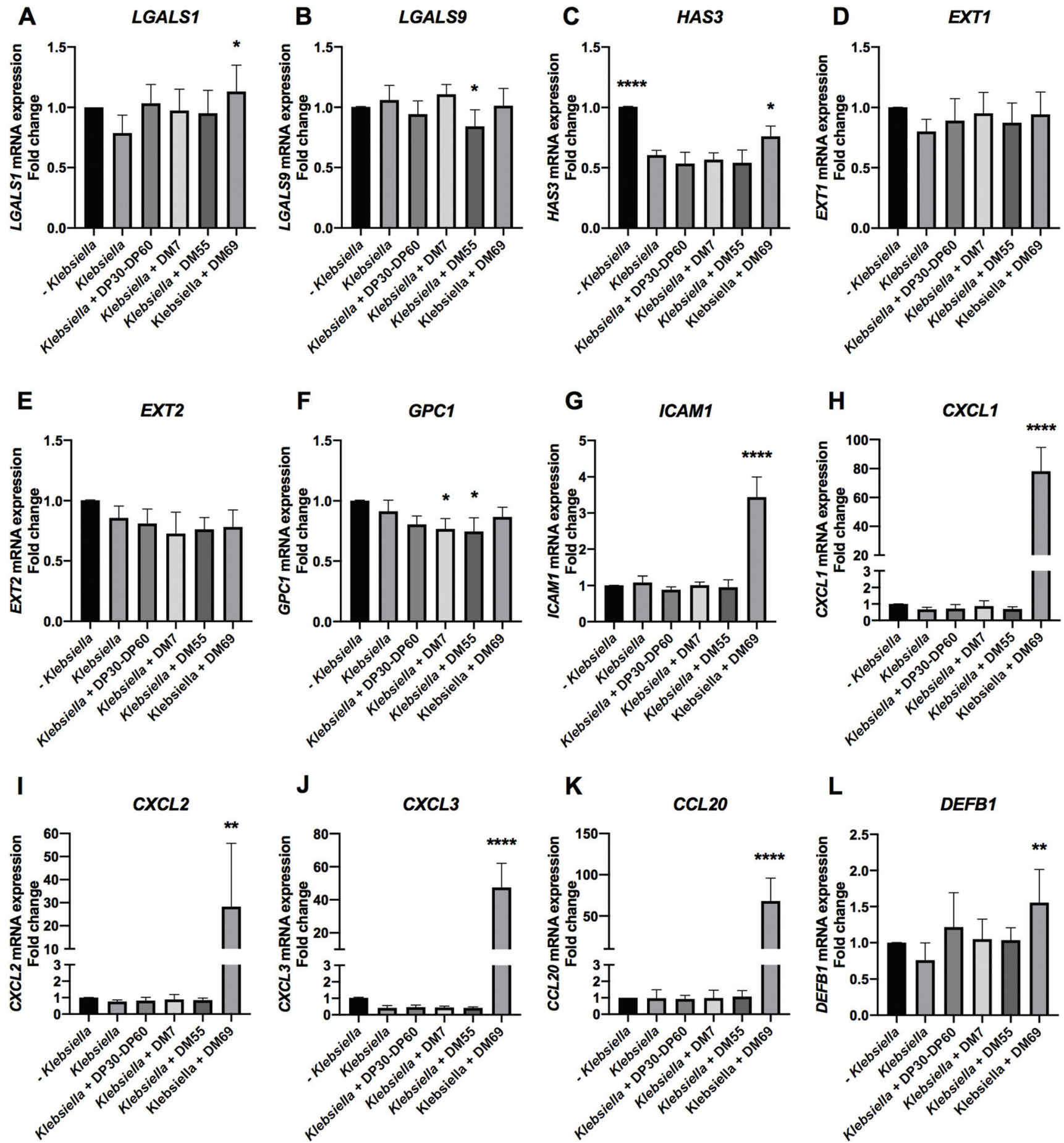

Fig. 11 K. pneumoniae LMG20218 infection and the effects of DP30-DP60 inulin and DM7, DM55, and DM69 pectins on the cell membrane glycosylation and inflammation genes in intestinal epithelial Caco-2 cells. Caco-2 cells were cultured for 21 days and pre-incubated with DP30-DP60 inulin and DM7, DM55, and DM69 pectins for $2 \mathrm{~h}$ before infection with $K$. pneumoniae LMG20218 harvested from the stationary phase. After infection, the cell membrane glycosylation genes [galectins (LGALS1 and LGALS9) and glycocalyx (HAS3, EXT1, EXT2 and GPC1)] and inflammation associated genes [cell adhesion and chemotaxis target genes (ICAM1, CXCL1, CXCL2, CXCL3 and CCL20) as well as the anti-microbial peptide gene (DEFB1)] were quantified by evaluating the mRNA expression with real-time RT-PCR. The results are presented as a fold change compared to the group without $K$. pneumoniae LMG20218 infection. Statistical analysis was performed using one-way ANOVA, comparing to the group only with K. pneumoniae LMG20218 infection, the group without $K$. pneumoniae LMG20218 infection and the group with pre-incubation of carbohydrates prior to K. pneumoniae LMG20218 infection ( ${ }^{*} p<0.05,{ }^{* *} p<0.01$ and ${ }^{* * * *} p<0.0001$ ). 
pathogens from different growth phases. ${ }^{26}$ This suggests that mixtures of inulins might be necessary to prevent pathogen infections in infant formula.

2 '-FL and inulins are the most widely applied hMO and NDC in infant formula, and both belong to the family of neutral oligosaccharides. Although many health benefits have been shown in clinical trials for these molecules, ${ }^{45,46}$ they still do not fully mimic the functions of hMOs in mother's milk that also contains acidic oligosaccharides. ${ }^{1}$ It has been suggested that acidic oligosaccharides are important for specific health benefits. ${ }^{1}$ Such an alternative acidic NDC substitute for hMO might be pectin that contains galacturonic acid and provides negatively charged carboxyl groups similar to what can be found on acidic sialylated hMO. ${ }^{1,47}$ Here, pectins with DM7, DM55, and DM69 all modulated gut epithelial cells, such that both gut pathogens and the lung pathogen had much less ability to adhere to the epithelial cells. This may suggest that for the anti-pathogenic effects of pectins, their negatively charged carboxyl groups may be a valuable addition for infant formula. ${ }^{48}$

The DM55 and DM69 pectins may govern the anti-adhesion effects on pathogens through modulation of cell membrane glycosylation-associated galectins. Our data demonstrate that pre-incubating Caco-2 cells with both DM55 and DM69 pectins inhibited the adhesion of $K$. pneumoniae LMG20218 from the stationary phase, but differently modulated the cell membrane glycosylation-associated galectin genes LGALS1 and LGASL9, which are responsible for the synthesis of galectin-1 and galectin-9, respectively. Galectins are a family of conserved glycanbinding proteins. ${ }^{14}$ Galectin-1 is widely distributed on epithelial cells in the small intestine and is downregulated in celiac disease ${ }^{14}$ Here, we show pre-incubating Caco-2 cells with DM69 pectin increased LGALS1 expression after the exposure of epithelial cells to $K$. pneumoniae LMG20218. This finding suggests a possible rescuing effect of DM69 pectin on the maintenance of gut homeostasis during infection. ${ }^{14}$ Galectin-9 is different from galectin-1 and highly expressed in patients with food allergy and considered to be a target for preventing allergic diseases. ${ }^{16}$ Here, we show that through pre-incubating Caco-2 cells with DM55 pectin, LGALS9 was downregulated after exposure to K. pneumoniae LMG20218, which may suggest an inflammation suppressive effect of DM55 pectin.

The DM7, DM55, and DM69 pectins may also govern the anti-adhesion effects of pathogens through modulation of glycocalyx-associated genes in epithelial cells. Pre-incubating Caco-2 cells with DM7, DM55, and DM69 pectins inhibited the adhesion of $K$. pneumoniae LMG20218 from the log phase (DM7 and DM55 pectins), $K$. pneumoniae LMG20218 from the stationary phase (DM7, DM55 and DM69 pectins), and E. coli LMG5862 from the log phase (DM69 pectin). This was associated with the downregulation of the epithelial glycocalyxassociated genes including HAS3, EXT1, and GPC1. In particular, $H A S 3$, the hyaluronic acid synthase gene, was significantly downregulated by all of the tested pathogenic strains and this was observed with pathogens from both $\log$ and stationary phases. HAS is responsible for the synthesis of HA, which is a highly viscous component of the intestinal epithelial glycocalyx glycosaminoglycan side chain and is responsible for tissue repair and anti-inflammatory effects. ${ }^{49}$ HAS3 downregulation may lead to the lower production of HA, resulting in a thinner glycocalyx barrier, which may increase the chances for pathogen infection. However, DM7 and DM69 pectin pre-incubation significantly upregulated $H A S 3$, which may enhance the barrier function of Caco-2 cells ${ }^{49}$ and reduce pathogen adhesion as we demonstrate for E. coli LMG5862 from the log phase and $K$. pneumoniae LMG20218 from both $\log$ and stationary phases.

Another glycocalyx gene that was downregulated by the exposure to pathogens was EXT1. EXT1 is involved in the elongation of one important glycocalyx molecule, which is the glycosaminoglycan side chain heparan sulfate (HS). HS supports the organogenesis, maintains gut barrier integrity, and can be cleaved by gut bacterial enzymes. ${ }^{50}$ EXT1 was downregulated in response to the exposure of $K$. pneumoniae LMG20218 from the log phase, but pre-incubating Caco-2 cells with DM7 pectin prevented this lowering of EXT1 expression. Moreover, DM7 and DM69 pectins upregulated the glycosaminoglycan chains HA and HS. This was different for DM55 pectin, which did not influence HA and HS, but induced a downregulation of the protein backbone glypican 1 (GPC1). DM7 pectin also induced a downregulation of GPC1. GPC1 acts as a carrier for the glycosaminoglycan chains in the glycocalyx layer. $^{51}$ Through the downregulation of GPC1, DM7 and DM55 pectins may modify the composition of the glycocalyx layer that provides binding sites for $K$. pneumoniae LMG20218. Consequently, DM7 and DM55 pectin pretreatment may reduce the adhesion of $K$. pneumoniae LMG20218 to epithelial cells, as we also found in our study. Overall, our data show that genes involved in glycocalyx synthesis are downregulated with pathogen infection, but specific pectins with DM7, DM55, and DM69 may prevent this by re-modeling the glycosaminoglycan side chains and the protein backbone of the glycocalyx and by that inhibit pathogen adhesion.

We observed a pathogen strain dependent effect on inflammation-associated genes and a structure dependent effect of pectins on restoring the expression of these genes. With the exception of E. coli LMG5862 and E. coli WA321, other tested gut pathogens induced upregulation of the cell adhesion and chemotaxis genes ICAM1, CXCL1, CXCL2, CXCL3, or CCL20. These genes were reported to be associated with Crohn's disease and ulcers. ${ }^{52}$ Even though E. coli LMG5862 alone did not influence these genes in Caco-2 cells, DM69 pectin pre-incubation dramatically upregulated ICAM1, CXCL1, CXCL3, and CCL20, which may suggest that DM69 pectin induced the facilitation of epithelial responses to E. coli LMG5862. For E. coli WA321, an enhanced epithelial attachment was measured after DM69 pectin pre-incubation, suggesting that DM69 pectin may facilitate the adhesion of E. coli WA321 to Caco-2 cells. Effects of other pathogens on the expression of inflammation-associated genes were however not changed by pectin pre-incubation.

Different from the gut pathogens, the lung pathogen K. pneumoniae LMG20218 induced a lowering effect on inflam- 
mation related genes. K. pneumoniae LMG20218 from the log phase downregulated ICAM1. A different interaction of K. pneumoniae LMG20218 with the host epithelium may suggest a different infection strategy compared to the gut pathogens mentioned above. This might be related to the fact that $K$. pneumoniae evolved with stronger resistance to antibiotic treatment, and a different biosynthesis and transportation pathway for virulence factors such as lipopolysaccharides. ${ }^{53}$ Interestingly, pre-incubation with DM7 and DM55 pectins restored the downregulation of ICAM1 induced by K. pneumoniae LMG20218 from the log phase. The upregulation of the inflammation genes might imply more production of proinflammatory cytokines and anti-microbial peptides, ${ }^{20,54}$ which might lead to the lower $K$. pneumoniae LMG20218 adhesion to Caco- 2 cells as we observed. This is also in line with the established assumption that pro-inflammatory signaling is crucial for K. pneumoniae LMG20218 clearance. $^{55}$

\section{Conclusions}

In summary, we demonstrate that 2 '-FL as well as pectins may prevent the adhesion of pathogens through modulating glycosylation genes of galectin and glycocalyx, and inflammatory genes in gut epithelial cells. The effects on this epithelium-pathogen interaction are structure-dependent. In particular, low DM pectin with DM7 have such an effect, while high DM pectins with DM55 and DM69 may also have a risk of modulating epithelial cells as such that it leads to increased adhesion of specific pathogens. Inulins probably exert anti-pathogenic effects through its wellknown effect on enhancing barrier function in gut epithelial cells. ${ }^{43}$ We also showed that pathogens themselves modulate gene expression in gut epithelial cells. Specific NDC molecules, i.e. DM69 pectin, upregulated inflammation-associated genes when gut epithelial cells were exposed to pathogens, which may suggest that DM69 pectin has an immune activating effect and by that contributes to the clearance of pathogen adhesion. ${ }^{55}$ This information can be used in the design of tailored infant formula with anti-adhesion properties on pathogens.

\section{Author contributions}

C.K. and P.d.V. conceived and designed the experiments. C.K., M.W., and B.J.d.H. performed the experiments. C.K. and B.J.d. H. analyzed the data. C.K., M.B., and P.d.V. wrote the paper.

\section{Conflicts of interest}

There are no conflicts to declare.

\section{Acknowledgements}

CK is supported by the China Scholarship Council (CSC) under Grant No. 201600090212.

\section{References}

1 L. Bode and E. Jantscher-Krenn, Structure-function relationships of human milk oligosaccharides, Adv. Nutr., 2012, 3, 383S.

2 K. P. Gopalakrishna and T. W. Hand, Influence of maternal milk on the neonatal intestinal microbiome, Nutrients, 2020, 12, 823.

3 C. R. Martin, P. R. Ling and G. L. Blackburn, Review of infant feeding: Key features of breast milk and infant formula, Nutrients, 2016, 8, 279.

$4 \mathrm{H}$. Hao, L. Zhu and H. S. Faden, The milk-based diet of infancy and the gut microbiome, Gastroenterol. Rep., 2019, 7, 246.

5 R. Akkerman, M. M. Faas and P. de Vos, Non-digestible carbohydrates in infant formula as substitution for human milk oligosaccharide functions: Effects on microbiota and gut maturation, Crit. Rev. Food Sci. Nutr., 2019, 59, 1486.

6 C. Dupont and Y. Vandenplas, Different thickening complexes with pectin in infant anti-regurgitation formula, Acta Paediatr., 2020, 109, 471.

7 C. Kong, M. M. Faas, P. De Vos and R. Akkerman, Impact of dietary fibers in infant formulas on gut microbiota and the intestinal immune barrier, Food Funct., 2020, 11, 9445.

8 R. Y. Wu, B. Li, Y. Koike, P. Määttänen, H. Miyake, M. Cadete, et al., Human Milk Oligosaccharides Increase Mucin Expression in Experimental Necrotizing Enterocolitis, Mol. Nutr. Food Res., 2019, 63, e1800658.

9 L. Cheng, C. Kong, M. T. C. Walvoort, M. M. Faas and P. de Vos, Human Milk Oligosaccharides Differently Modulate Goblet Cells Under Homeostatic, Proinflammatory Conditions and ER Stress, Mol. Nutr. Food Res., 2019, 64, e1900976.

10 N. Arabyan, D. Park, S. Foutouhi, A. M. Weis, B. C. Huang, C. C. Williams, et al., Salmonella Degrades the Host Glycocalyx Leading to Altered Infection and Glycan Remodeling, Sci. Rep., 2016, 8, 29525.

11 Y. Yu, Y. Chen, P. Mikael, F. Zhang, A. M. Stalcup, R. German, et al., Surprising absence of heparin in the intestinal mucosa of baby pigs, Glycobiology, 2017, 27, 57.

12 G. Patsos and A. Corfield, Management of the human mucosal defensive barrier: Evidence for glycan legislation, Biol. Chem., 2009, 390, 581.

13 C. L. Romanò, E. De Vecchi, M. Bortolin, I. Morelli and L. Drago, Hyaluronic Acid and Its Composites as a Local Antimicrobial/Antiadhesive Barrier, J. Bone Jt Infect., 2017, $2,63$.

14 V. Sundblad, A. A. Quintar, L. G. Morosi, S. I. Niveloni, A. Cabanne, E. Smecuol, et al., Galectins in intestinal inflammation: Galectin-1 expression delineates response to treatment in celiac disease patients, Front. Immunol., 2018, 9, 379 .

15 G. A. Rabinovich, Y. van Kooyk and B. A. Cobb, Glycobiology of immune responses, Ann. N. Y. Acad. Sci., 2012, 1253, 1. 
16 S. De Kivit, A. D. Kraneveld, L. M. G. Knippels, Y. van Kooyk, J. Garssen and L. E. M. Willemsen, Intestinal epithelium-derived galectin- 9 is involved in the immunomodulating effects of nondigestible oligosaccharides, J. Innate Immun., 2013, 5, 625.

17 K. Bandow, J. Kusuyama, M. Shamoto, K. Kakimoto, T. Ohnishi and T. Matsuguchi, LPS-induced chemokine expression in both MyD88-dependent and -independent manners is regulated by Cot/Tpl2-ERK axis in macrophages, FEBS Lett., 2012, 586, 1540.

18 N. Müller, The role of intercellular adhesion molecule-1 in the pathogenesis of psychiatric disorders, Front. Pharmacol., 2019, 10, 1251.

19 D. E. Iglesias, E. Cremonini, C. G. Fraga and P. I. Oteiza, Ellagic acid protects Caco-2 cell monolayers against inflammation-induced permeabilization, Free Radicals Biol. Med., 2020, 152, 776.

20 J. Raschig, D. Mailänder-Sánchez, A. Berscheid, J. Berger, A. A. Strömstedt, L. F. Courth, et al., Ubiquitously expressed Human Beta Defensin 1 (hBD1) forms bacteria-entrapping nets in a redox dependent mode of action, PLoS Pathog., 2017, 13, e1006261.

21 J. Lu, M. Pierce, A. Franklin, T. Jilling, D. M. Stafforini and M. Caplan, Dual Roles of Endogenous Platelet-activating Factor Acetylhydrolase in a Murine Model of Necrotizing Enterocolitis, Pediatr. Res., 2010, 68, 225.

22 C. L. R. Belmiro, H. S. P. Souza, C. C. S. Elia, M. T. L. Castelo-Branco, F. R. Silva, R. L. Machado, et al., Biochemical and immunohistochemical analysis of glycosaminoglycans in inflamed and non-inflamed intestinal mucosa of patients with Crohn's disease, Int. J. Colorectal Dis., 2005, 20, 295.

23 C. Berne, C. K. Ellison, A. Ducret and Y. V. Brun, Bacterial adhesion at the single-cell level, Nat. Rev. Microbiol., 2018, 16, 616.

24 J. Jaishankar and P. Srivastava, Molecular basis of stationary phase survival and applications, Front. Microbiol., 2017, 8, 2000.

25 D. E. Chang, D. J. Smalley and T. Conway, Gene expression profiling of Escherichia coli growth transitions: An expanded stringent response model, Mol. Microbiol., 2002, 45, 289.

26 R. L. Bertranda, Lag phase is a dynamic, organized, adaptive, and evolvable period that prepares bacteria for cell division, J. Bacteriol., 2019, 201, e00697.

27 B. Herigstad, M. Hamilton and J. Heersink, How to optimize the drop plate method for enumerating bacteria, J. Microbiol. Methods, 2001, 44, 121.

28 V. L. Thijssen, S. Hulsmans and A. W. Griffioen, The galectin profile of the endothelium: Altered expression and localization in activated and tumor endothelial cells, Am. J. Pathol., 2008, 172, 545.

29 Z. Zhang, Z. Tang, X. Ma, K. Sun, L. Fan, J. Fang, et al., Correction to: TAOK1 negatively regulates IL-17-mediated signaling and inflammation, Cell. Mol. Immunol., 2018, 15, 940.
30 K. Feuser, K. P. Thon, S. C. Bischoff and A. Lorentz, Human intestinal mast cells are a potent source of multiple chemokines, Cytokine, 2012, 58, 178.

31 C. Kong, L. Cheng, G. Krenning, J. Fledderus, B. J. de Haan, M. T. C. Walvoort, et al., Human milk oligosaccharides mediate the crosstalk between intestinal epithelial Caco-2 cells and lactobacillus plantarum WCFS1 in an in vitro model with intestinal peristaltic shear force, J. Nutr., 2020, 15, 2077.

32 A. C. R. Nahás-Scocat, G. F. A. de Moraes, H. B. Nader, C. M. Vicente and L. Toma, Analysis of proteoglycan expression in human dental pulp, Arch. Oral Biol., 2018, 90, 67.

33 E. Kim, K. Hwang, J. Lee, S. Y. Han, E. M. Ki, J. Park, et al., Skin protective effect of epigallocatechin gallate, Int. J. Mol. Sci., 2018, 19, 173.

34 X. Guo, M. Lin, W. Yan, W. Chen and G. Hong, A novel splice mutation induces exon skipping of the EXT1 gene in patients with hereditary multiple exostoses, Int. J. Oncol., 2019, 54, 859.

35 R. Lu, X. Zeng, Q. Han, M. Lin, L. Long, H. Dan, et al., Overexpression and selectively regulatory roles of IL-23/ IL-17 axis in the lesions of oral lichen planus, Mediators Inflamm., 2014, 2014, 701094.

36 L. M. Ashander, B. Appukuttan, Y. Ma, D. Gardner-Stephen and J. R. Smith, Targeting Endothelial Adhesion Molecule Transcription for Treatment of Inflammatory Disease: A Proof-of-Concept Study, Mediators Inflamm., 2016, 2016, 7945848.

37 M. S. Thiese, B. Ronna and U. Ott, P value interpretations and considerations, J Thorac. Dis., 2016, 8, e928.

38 L. Cheng, C. Kong, M. T. C. Walvoort, M. M. Faas and P. de Vos, Human Milk Oligosaccharides Differently Modulate Goblet Cells Under Homeostatic, Proinflammatory Conditions and ER Stress, Mol. Nutr. Food Res., 2020, 64, e1900976.

39 M. Nishida, K. Murata, K. Oshima, C. Itoh, K. Kitaguchi, Y. Kanamaru, et al., Pectin from Prunus domestica L. induces proliferation of IEC-6 cells through the alteration of cell-surface heparan sulfate on differentiated Caco-2 cells in co-culture, Glycoconjugate J., 2015, 32, 153.

40 K. Chen, H. Chen, M. M. Faas, B. J. de Haan, J. Li, P. Xiao, et al., Specific inulin-type fructan fibers protect against autoimmune diabetes by modulating gut immunity, barrier function, and microbiota homeostasis, Mol. Nutr. Food Res., 2017, 61, 1601006.

41 M. Piotrowski, D. Wultańska, P. Obuch-Woszczatyński and H. Pituch, Fructooligosaccharides and mannose affect Clostridium difficile adhesion and biofilm formation in a concentration-dependent manner, Eur. J. Clin. Microbiol. Infect. Dis., 2019, 38, 1975.

42 G. A. Sprenger, F. Baumgärtner and C. Albermann, Production of human milk oligosaccharides by enzymatic and whole-cell microbial biotransformations, J. Biotechnol., 2017, 258, 79. 
43 J. Song, Q. Li, N. Everaert, R. Liu, M. Zheng, G. Zhao, et al., Effects of inulin supplementation on intestinal barrier function and immunity in specific pathogen-free chickens with Salmonella infection, J. Anim. Sci., 2020, 98, skz396.

44 S. L. Walker, J. E. Hill, J. A. Redman and M. Elimelech, Influence of growth phase on adhesion kinetics of Escherichia coli D21 g, Appl. Environ. Microbiol., 2005, 71, 3093.

45 K. C. Goehring, B. J. Marriage, J. S. Oliver, J. A. Wilder, E. G. Barrett and R. H. Buck, Similar to Those Who Are Breastfed, Infants Fed a Formula Containing 2'Fucosyllactose Have Lower Inflammatory Cytokines in a Randomized Controlled Trial, J. Nutr., 2016, 146, 2559.

46 H. Oswari, A. D. Widodo, F. Handayani, M. Juffrie, T. Sundjaya, J. Bindels, et al., Dosage-related prebiotic effects of inulin in formula-fed infants, Pediatr. Gastroenterol. Hepatol. Nutr., 2019, 22, 63.

47 Y. Sun, Y. He, F. Wang, H. Zhang, P. de Vos and J. Sun, Low-methoxyl lemon pectin attenuates inflammatory responses and improves intestinal barrier integrity in caerulein-induced experimental acute pancreatitis, Mol. Nutr. Food Res., 2017, 61, 1.

48 J. P. Van Den Berg, E. A. M. Westerbeek, F. R. M. van der Klis, E. A. M. Sanders, G. A. M. Berbers and R. M. van Elburg, Response on Pneumococcal Vaccine in Preterm Infants After Neutral and Acidic Oligosaccharides Supplementation, Pediatr. Infect. Dis. J., 2015, 34, 976.
49 N. Joksimovic, G. Spasovski, V. Joksimovic, V. Andreevski, C. Zuccari and C. F. Omini, Efficacy and tolerability of hyaluronic acid, tea tree oil and methyl-sulfonyl-methane in a new gel medical device for treatment of haemorrhoids in a double-blind, placebo-controlled clinical trial, Updates Surg., 2012, 64, 195.

50 M. Busse and M. Kusche-Gullberg, In Vitro Polymerization of Heparan Sulfate Backbone by the EXT Proteins, J. Biol. Chem., 2003, 278, 41333.

51 H. Kang, Y. Fan, P. Zhao, C. Ren, Z. Wang and X. Deng, Regional specific modulation of the glycocalyx and smooth muscle cell contractile apparatus in conduit arteries of tailsuspended rats, J. Appl. Physiol., 2016, 120, 537.

52 L. Gillberg, M. Varsanyi, M. Sjöström, M. Lördal, J. Lindholm and P. M. Hellström, Nitric oxide pathwayrelated gene alterations in inflammatory bowel disease, Scand. J. Gastroenterol., 2012, 47, 1283.

53 L. P. P. Patro and T. Rathinavelan, Targeting the Sugary Armor of Klebsiella Species, Front. Cell. Infect. Microbiol., 2019, 9, 367.

54 S. Wickramasinghe, A. R. Pacheco, D. G. Lemay and D. A. Mills, Bifidobacteria grown on human milk oligosaccharides downregulate the expression of inflammationrelated genes in Caco-2 cells, BMC Microbiol., 2015, 15, 172.

55 J. A. Bengoechea and J. Sa Pessoa, Klebsiella pneumoniae infection biology: Living to counteract host defences, FEMS Microbiol. Rev., 2019, 43, 123. 